Ecología Austral 31:148-169 Abril 2021

Asociación Argentina de Ecología

https://doi.org/10.25260/EA.21.31.1.0.1096

\title{
Dietas saludables y sustentables, ¿Son posibles en la Argentina?
}

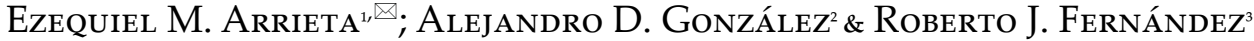

${ }^{1}$ Instituto Multidisciplinario de Biología Vegetal, CONICET - Universidad Nacional de Córdoba. Córdoba, Argentina. ${ }_{2}^{2}$ Instituto Andino-Patagónico de Tecnologías Biológicas y Geoambientales, CONICET - Universidad Nacional del Comahue. San Carlos de Bariloche, Río Negro, Argentina. ${ }^{3}$ Cátedra de Ecología e IFEVA-CONICET, Facultad de Agronomía, Universidad de Buenos Aires. Ciudad Autónoma de Buenos Aires, Argentina.

\begin{abstract}
Resumen. Para afrontar el reto de alimentar a una población creciente que cambia su estilo de vida y su dieta $\mathrm{y}$, al mismo tiempo, tratar de conservar los recursos naturales y proteger la biodiversidad, se proponen tres estrategias principales para lograr tales objetivos: 1) cambiar la forma de producir los alimentos en el campo (aumentando la eficiencia), 2) reducir las pérdidas y desperdicios a lo largo de la cadena agroalimentaria, y 3) promover la adopción de dietas saludables y sostenibles. Las dos primeras estrategias fueron contempladas en nuestro país y forman parte de la agenda académica, gubernamental y productiva, pero la tercera ha sido muy poco explorada. A través de un análisis de la bibliografía destacamos la importancia de incluir a las dietas saludables como herramienta para contribuir simultáneamente a la sustentabilidad del sistema agroalimentario y al mejoramiento de la salud pública, y resaltamos los desafíos que esto implicaría para el abastecimiento del mercado interno. Para ello, primero presentamos información de los impactos ambientales de los sistemas agroalimentarios y las dos primeras estrategias nombradas, utilizadas tradicionalmente para abordarlos. Segundo, exponemos los motivos por los cuales la búsqueda de la eficiencia no debería ser el único camino a seguir debido a las consecuencias esperadas del cada vez mejor conocido ‘Efecto Rebote' (o Paradoja de Jevons). Tercero, analizamos las evidencias a favor de una correlación positiva entre los beneficios para la salud de varios grupos de alimentos (en general, de origen vegetal) y el impacto relativamente bajo que tiene su producción sobre el ambiente. Finalmente, hacemos un breve análisis físico (no económico) sobre la capacidad que tendría el sistema agroalimentario nacional para proveer de alimentos saludables en cantidad suficiente y sus consecuencias para el ambiente y el sector productivo.
\end{abstract}

[Palabras clave: sistemas agroalimentarios, salud pública, sostenibilidad, nutrición, enfermedades crónicas no-transmisibles, seguridad alimentaria]

Aвstract. Healthy and sustainable diets: Are possible in Argentina? To face the challenge of feeding a growing population that changes its life-style and diet while trying to conserve natural resources and to protect biodiversity, there are three main strategies to achieve such sustainability goals: 1) changing current agricultural practices, 2) reducing losses and waste throughout the food production and processing chain, and 3) promoting healthy and sustainable diets. Strategies (1) and (2) have been largely considered in Argentina and are part of the academic, government and industrial agenda, but the third strategy has been given little attention so far. By a revision and literature analysis, we show the relevance of healthy diets as a tool to increase the sustainability of the national food system and to improve public health, as well as the challenges it poses to domestic supply. First, we present information on the environmental impacts of food systems and the strategies traditionally used to increase sustainability: production efficiency, food losses and waste reduction. Second, we argue that efficiency should not be the only way for improvement because of the consequences that arise from rebound effects (a.k.a. Jevons paradox). Third, we analyze the evidence in favor of a positive correlation between the health benefits of several food groups (mostly plant-based foods) and their relatively low environmental impact. Finally, we briefly analyze the capacity of the national food system to provide enough food - physically, but not economically - to achieve healthier diets, and the implications that this would have on production and the environment.

[Keywords: food systems, public health, sustainability, nutrition, chronic noncommunicable diseases, food security, food sovereignty]

Editor asociado: Lucas Garibaldi

$\bar{\triangle}$ earrieta@imbiv.unc.edu.ar
Recibido: 30 de Diciembre de 2019

Aceptado: 10 de Noviembre de 2020 


\section{INTRODUCCIÓN}

Mitigar los problemas ambientales asociados a la producción de alimentos es un mantra que hemos estado recitando desde hace muchos años, aunque con diferente vocabulario y argumentos. Sin embargo, por más que construyamos modelos productivos capaces de proveer alimentos en cantidad suficiente y calidad adecuada, las elecciones de los consumidores pueden finalmente determinar la demanda de alimentos a mediano y largo plazo y, en consecuencia, el uso de los recursos naturales y el deterioro del ambiente (Lusk and McCluskey 2018; Poore and Nemecek 2018; Springmann et al. 2018; Leclère et al. 2020). A esto se suma que las elecciones alimentarias también afectan a la salud humana de manera significativa y, junto con el sedentarismo, son en gran parte responsables de la elevada prevalencia actual (y creciente) de las enfermedades crónicas notransmisibles (ECNT, ver más abajo) (Afshin et al. 2019). Mediante una revisión y un análisis de la bibliografía, en este artículo destacamos la importancia y la factibilidad de incluir a las dietas saludables como una herramienta para contribuir a la sostenibilidad del sistema agroalimentario de la Argentina, así como los desafíos y las consecuencias que tal decisión implicaría para el sector productivo.

\section{IMPACTOS SOBRE EL AMBIENTE DE LOS SISTEMAS AGROALIMENTARIOS GLOBAL Y NACIONAL}

A la vez que las hambrunas masivas fueron quedando atrás, la producción agropecuaria se convirtió en una de las actividades humanas con mayor impacto sobre la estructura y el funcionamiento de la biósfera (Dobermann et al. 2013; FAO 2017). En la actualidad, la humanidad usa casi la mitad de la superficie terrestre libre de hielo para producir alimentos, fibras y bioenergía (Foley et al. 2011): 3203 Mha de pasturas, 871 Mha de cultivos para consumo humano directo, 497 Mha de cultivos para consumo animal, 140 Mha de cultivos para biocombustibles, 33 Mha de cultivos para fibra y 16 Mha para otros usos (Alexander et al. 2016). Durante el siglo XX se transformaron más de mil millones de hectáreas de ecosistemas naturales en cultivos de cosecha y forrajeros (Bringezu et al. 2014), lo cual redujo la biodiversidad a niveles alarmantes (Díaz et al. 2019). Se estima que 30\% de las emisiones antropogénicas mundiales de gases de efecto invernadero (GEI) provienen del sistema agroalimentario, mientras que la aplicación masiva de fertilizantes está alterando los ciclos biogeoquímicos del nitrógeno y del fósforo de una manera que recién empezamos a comprender (Bouwman et al. 2009; Vermeulen et al. 2012). La aplicación excesiva de pesticidas en los cultivos contribuye a contaminar suelos y aguas, a diezmar las poblaciones de polinizadores y de otros insectos de los que depende parte de la producción, así como a dañar la salud humana y de los ecosistemas en general (Potts et al. 2016; Dainese et al. 2019). El uso de agua dulce, que en la actualidad irriga 300 millones de hectáreas, causó la salinización de los suelos y el vaciamiento de las reservas hídricas en muchas regiones del mundo. Pero los problemas no se limitan a los ecosistemas terrestres, ya que la explotación de los recursos marinos llevó a saturar o exceder la capacidad de pesca en el $90 \%$ de las zonas de pesca (Pauly and Zeller 2017). Además, el sistema agroalimentario demanda indirectamente recursos al incluir instalaciones y maquinaria (e.g., puertos, barcos, tractores, cosechadoras, alambrados, silos) elaboradas con metales, maderas, plásticos y otros insumos de origen industrial cuya manufactura demanda combustibles fósiles (Pelletier et al. 2011; Hajer et al. 2016; Schramski et al. 2020). A lo anterior se le suma una gran diversidad de sustancias químicas, que incluye los antibióticos y otras muy usadas para promover el crecimiento de los animales domésticos y para tratar y prevenir enfermedades cuando se los cría en condiciones de hacinamiento (van Boeckel et al. 2015; van Boeckel et al. 2019). Además de la dimensión ecológica, la esfera social también se vio afectada por conflictos rurales debido a las consecuencias negativas del modelo productivo para muchas comunidades campesinas, pobladores rurales y pueblos originarios, como el desplazamiento y la pérdida de medios de vida (García-López and Arizpe 2010; Cáceres 2015). A esto se le agrega que la reciente pandemia de enfermedad por coronavirus demostró la fragilidad del sistema alimentario y la cara invisibilizada de la severa tendencia a la urbanización y el predominio de unas pocas cadenas de logística y comercialización de alimentos (Echeverría 2020).

El aumento esperado en el tamaño de la población global y los cambios en las dietas - traccionados sobre todo por los mayores ingresos per cápita en los países en vías de desarrollo- han determinado (y lo seguirán 
haciendo) un aumento en la demanda de alimentos tanto en cantidad como en calidad que puede agravar sensiblemente el impacto ambiental de los sistemas agroalimentarios globales y nacionales si no se actúa en consecuencia (Tilman et al. 2011; FAO 2018). Springmann et al. (2018) estimaron que para el año 2050 la producción mundial de alimentos, en un escenario en el cual las cosas continúan sin mayores cambios (business as usual), deberá incrementarse un $70 \%$ respecto a la del año 2010. De ser así, los impactos ambientales serían considerables: la superficie de cultivos debería pasar de 12 a 21 millones de $\mathrm{km}^{2}$ (con 100-200 millones de hectáreas adicionales deforestadas en África Subsahariana y Latinoamérica), las emisiones anuales de GEI de 5.2 a 9.9 millones de toneladas de dióxido de carbono equivalente, la extracción anual de agua dulce de 1800 a $2970 \mathrm{~km}^{3}$, mientras que la aplicación de fertilizantes debería ser $51 \%$ y $54 \%$ mayor que en el presente para el nitrógeno y el fósforo, respectivamente. Aquí resulta necesario recalcar que varios autores coinciden en que la producción actual de alimentos en el mundo ya es suficiente para proveer las calorías necesarias para alimentar a la población entera, e incluso quizá también a la proyectada para el 2050 (McIntyre et al. 2009; Tilman et al. 2011; Holt-Giménez et al. 2012). Claramente, lo que es necesario cambiar con respecto al escenario de business as usual es la inequidad en el acceso a la comida, y la visión de las commodities como un producto comercial más, sin considerar su dimensión como alimento (Fernández 2015).

En la Argentina ocurre una particularidad: mientras que muchas prácticas agrícolas son similares a las llevadas a cabo en muchos otros países alcanzados por la Revolución Verde, las consecuencias ambientales observadas en las últimas décadas están representadas tanto por los impactos ambientales asociados a la agricultura y ganadería intensivas de los países de altos ingresos (eutrofización y toxicidad química) como a los de la agricultura y ganadería más extensiva de los países de bajos ingresos (aumento de la superficie agropecuaria y deforestación) (Viglizzo et al. 2011; Pellegrini and Fernández 2018). Dehecho, el avance de la frontera productiva a costa de ecosistemas naturales y seminaturales, con las consecuentes pérdidas de biodiversidad y servicios ecosistémicos, constituye uno de los problemas ambientales más importantes del país (MAyDS 2020). Además, la aplicación de pesticidas en frecuencias y dosis crecientes es una preocupación palpable en la población general y en la comunidad médica y académica (Arrieta et al. 2019). En este sentido, el rol autoproclamado por la Argentina como 'granero' o 'supermercado' del mundo hace que los sucesivos gobiernos formulen nuestras políticas agrarias en torno a las proyecciones de aumento en la demanda planetaria de alimentos y las posibles oportunidades del mercado, con poca ( $\mathrm{y}$ a veces nula) atención al deterioro ambiental. Esto se puede observar claramente en el Plan Estratégico Agroalimentario y Agroindustrial Argentina 2010-2020 (Felcman 2013), que propuso metas orientadas a mantener los incrementos de producción que ya se venían dando en función de la demanda global - muchas de las cuales se cumplieron - , aunque eso generó impactos ambientales negativos como la reducción de la superficie de bosques nativos y otras áreas naturales, conflictos territoriales, expulsión de comunidades, etc. (Silvetti et al. 2013; Cáceres 2015).

Aunque cumplir con la meta de abastecer de alimentos a la creciente población mundial representa un desafío complejo y ambicioso, existe consenso en que es posible hacerlo de forma sostenible (Chaudhary et al. 2018; Springmann et al. 2018; Willet et al. 2019). Sin embargo, llevar a los sistemas agroalimentarios global y nacional en esa dirección requiere de múltiples y diversas acciones (y no excluyentes), que pueden clasificarse en tres grupos (Keating et al. 2014; Fraser et a. 2016; Alexander et al. 2019): 1) cambios en las tecnologías y técnicas empleadas en la producción, 2) reducciones de las pérdidas y desperdicios en la cadena alimentaria, y 3) modificaciones en la demanda de alimentos (tanto en cantidad como en calidad) por parte de los consumidores. Si bien reconocemos que los sistemas alimentarios están compuestos por un gran número de actores sociales cuya interacción incrementa la complejidad (e.g., procesadores, industrias, distribuidores, comercializadores, etc.), en las secciones siguientes nos limitaremos a desarrollar algunos aspectos relacionados con estas tres estrategias.

\section{LA EFICIENCIA COMO CAMINO PARA REDUCIR EL IMPACTO AMBIENTAL}

Las soluciones propuestas para reducir el impacto de la producción agropecuaria se han basado tradicionalmente en incrementar la eficiencia a través de cambios tecnológicos y 
técnicos, sobre todo apuntando a reducir el uso de recursos por unidad de producto y a generar un desacople entre el crecimiento económico/ productivo y el deterioro ambiental (Parrique et al. 2019). Así, la agricultura industrial tal cual la conocemos (hija de la Revolución Verde) está evolucionando hacia una versión mejorada de ella misma con el objetivo de resolver los problemas causados por los errores del pasado: la intensificación sustentable (Godfray and Garnett 2014). Ésta, al mismo tiempo que intenta reducir el impacto ambiental por unidad de producto mediante el uso eficiente y racional de los insumos, busca producir más alimentos sin aumentar la superficie de tierra utilizada (Tilman et al. 2011). Sin embargo, la intensificación sustentable continúa con la tendencia de utilización de máquinas e insumos basados principalmente en el petróleo, y no parece cuestionar el destino que pueda tener lo que se produce (como materia prima de alimentos ultraprocesados, alimento para animales domésticos, $\mathrm{u}$ otros fines como los biocombustibles), en particular porque hasta el momento los esfuerzos se enfocaron en incrementar la eficiencia productiva de los commodities (Fernández et al. 2019). Dado que la agricultura industrial y la industria alimentaria se han moldeado entre sí, el camino ofrecido por la agricultura industrial y la intensificación sustentable está representado por una agricultura altamente especializada y tecnificada, en combinación con sistemas comerciales que funcionan ágilmente y permiten a los consumidores tener acceso a una variedad de alimentos diferentes en casi cualquier lugar (Aguiar et al. 2020).

Como alternativa a la agricultura industrial, y su conversión hacia la intensificación sustentable, se han propuesto a la agroecología y la intensificación ecológica, que de acuerdo a Gliessman (2007) consisten en "[...] aplicar conceptos y principios ecológicos al diseño y la gestión de sistemas alimentarios sostenibles", y así reducir (y algunos afirman que hasta eliminar) la necesidad de utilizar insumos externos en las prácticas agrícolas y ganaderas (Tittonell 2014; Wezel et al. 2015; Garibaldi et al. 2019). De esta manera, las prácticas aplicadas a escala de lote y de paisaje aumentarían la oferta de servicios ecosistémicos además de proveer bienes de aprovisionamiento como alimentos y fibras (Liere et al. 2017; Fernández et al. 2019). No es propósito de este artículo profundizar en el concepto de agroecología, pero cabe mencionar que ésta también incluye dentro de su marco conceptual a los problemas del sistema alimentario que ocurren por fuera del establecimiento agropecuario, lo que la ha convertido por eso además en un movimiento socio-político.

Desde la perspectiva productiva, las alternativas a la agricultura industrial se enfocan principalmente en aumentar la eficiencia en la fase de producción o 'hasta la tranquera'. Esta aproximación, que si bien es importante, presenta una limitación fundamental: una fracción de lo producido se pierde en el camino hacia la mesa del consumidor (Gustavsson et al. 2011). Esto toma aún más relevancia cuando se nota que, junto a esta pérdida de alimentos, también se pierden y desperdician fertilizantes, agua, combustibles, pesticidas y otros insumos usados para producirlos, aumentando su 'huella' (Swannell et al. 2019). Nuevamente, cabe mencionar que el movimiento agroecológico también propone acortar las cadenas de comercialización para aprovechar ésta y otras ventajas del acercamiento entre productores y consumidores, como el apoyo a la agricultura familiar y el desarrollo rural. Poniéndolo en números, se estima que a escala global se pierden a lo largo de la cadena agroindustrial y se desperdician en el hogar alrededor de $30 \%$ de los alimentos producidos, aunque con proporciones diferentes dependiendo del país, la región y la cadena alimentaria que se trate. En ese sentido, se observa una mayor pérdida (en el lote y durante el transporte) en los países de bajos y medianos ingresos, mientras que el desperdicio en el hogar y en los restaurantes es mayor en los países de ingresos altos (Gustavsson et al. 2011; Alexander et al. 2019). La cadena de suministro de frutas y verduras es la que presenta las mayores pérdidas y desperdicios. Uno de los motivos por lo que sucede esto último, es que la mercadería no cumple con los estándares de calidad y estética del mercado - en parte por consumidores desinformados o influenciados por una narrativa de cómo deben ser las frutas y verduras - a pesar de encontrarse en perfectas condiciones para consumo humano (por ejemplo, frutas con manchas negras superficiales inocuas). En contraposición con las estimaciones globales, el Programa Nacional de Reducción de Pérdidas y Desperdicios desarrolló un análisis con el objetivo de cuantificar pérdidas y desperdicios en cinco cadenas de alimentos en la Argentina (carne vacuna, leche, girasol, trigo, tres hortalizas y manzana), encontrando que durante el año 2017 se perdieron unas 
14.5 millones de toneladas y desperdiciaron otras 1.5 millones de toneladas (Schein 2018 ), el $12.5 \%$ de la producción total, un valor menor al promedio mundial $(30 \%)$. Sin embargo, es altamente probable que la inclusión de los alimentos que presentan una mayor prevalencia de pérdidas en la cadena de suministro eleve dicho número (particularmente de frutas y verduras). Esto sugiere que existen oportunidades y vías para reducir las ineficiencias tanto 'tranqueras afuera' (e.g., educando a los consumidores, procesadores, fabricantes y transportistas) como 'tranqueras adentro' (e.g., mejorando la investigación y la extensión agropecuarias).

\section{El Efecto Rebote: Ser eficiente PUEDE SER CONTRAPRODUCENTE}

Lo expresado en la sección anterior nos podría llevar a concluir que aumentar la eficiencia es el mejor camino para reducir el impacto ambiental de las actividades humanas. Pero en muchas ocasiones, el supuesto ahorro de recursos naturales se ve más que contrarrestado por un aumento en el consumo de bienes y servicios por distintos motivos directos e indirectos como, por ejemplo, una reducción de los costos (Freeman et al. 2016). Este fenómeno es conocido como el Efecto Rebote, y fue originalmente descripto para la producción de energía a partir de carbón en Inglaterra a mediados del siglo XIX por William Jevons (de ahí que también se lo conozca como la Paradoja de Jevons), quien demostró que las mejoras en la eficiencia de las máquinas a vapor habían causado un aumento en el uso total de carbón en lugar de mantenerlo o reducirlo.

Existen múltiples ejemplos en la industria donde el aumento en la eficiencia tecnológica (definido como la cantidad de producto obtenido por unidad de energía u otro insumo o factor limitante) se vio acompañado de un incremento en la oferta del producto y en la demanda del insumo o del factor en cuestión. Hay ejemplos bien documentados en la industria del aluminio y del hierro, en el sector de generación eléctrica, y el transporte aéreo y terrestre (Dahmus 2014). Por ejemplo, un Efecto Rebote directo de los autos modernos es que, al ser los más eficientes de la historia y poder recorrer más kilómetros por unidad de combustible, han generado un aumento del uso total de combustible (Moshiri et al. 2017; Andersson et al. 2019). Por el otro lado, un efecto indirecto derivado del uso eficiente del combustible por parte de los autos, es el desarrollo de barrios suburbanos. En este caso, las personas viajan diariamente muchos kilómetros hacia su lugar de trabajo desde su hogar (casi siempre casas más grandes y energéticamente costosas de mantener), lo cual implica también la demanda de más y mejores rutas.

Un razonamiento usual en estudios de impacto es que las emisiones de un contaminante dado por parte de una acción humana (e.g., la producción de acero) están determinadas por dos componentes que se multiplican: el factor de emisión de esa acción (i.e., la cantidad de dióxido de carbono liberado en toda la cadena que lleva a producir una tonelada de acero) multiplicada por lo que se conoce como la actividad (en este caso, las toneladas de acero producidas por ese país $\mathrm{o}$ industria). Usando este vocabulario, lo que predice el Efecto Rebote es que el aumento de la actividad suele más que compensar las caídas en los factores de emisión logradas por el avance tecnológico (en este ejemplo, aumentando de todos modos la cantidad total de dióxido de carbono emitida a pesar de la disminución en la emisión de dióxido de carbono por tonelada de acero producido).

La agricultura y la ganadería no han escapado del Efecto Rebote causado por las mejoras tecnológicas. Como se puede observar en la Tabla 1, la duplicación de la eficiencia energética durante la manufactura de los fertilizantes nitrogenados durante el último medio siglo se vio acompañado por un aumento en nueve veces de su producción. De la misma manera, el aumento en la productividad animal (tanto en lo que respecta a carne, como leche y huevos), fue seguido de un aumento en la producción total, lo que revela que no sólo hemos aprendido a criar animales más productivos, sino que criamos muchos más (Thornton 2010). El caso de los granos es particularmente relevante: Norman Borlaug, padre de la Revolución Verde, predijo que a medida que los rendimientos aumentaran (en $\mathrm{kg} / \mathrm{ha}$ ), la superficie agrícola cultivada se reduciría, hipótesis conocida como de land sparing (ahorro de tierra). Como el rendimiento agrícola no es otra cosa que la eficiencia en el uso de la tierra, los principales cultivos siguieron la tendencia predicha por el Efecto Rebote y la superficie ocupada por esos cultivos también se incrementó a escala global (Pellegrini and Fernández 2018). Aun así, Norman Borlaug no se equivocó del todo. Se estima que el aumento de los rendimientos 
Tabla 1. Cambios en la eficiencia para producir un insumo agronómico (fertilizante nitrogenado) y productividad de cuatro productos de origen animal, y tres de origen vegetal durante el período de expansión mundial de la Revolución Verde.

Table 1. Changes in efficiency to produce one agronomic input (nitrogen fertilizer) and productivity of four animal products and three plants during the expansion period of the Green Revolution.

\begin{tabular}{|c|c|c|c|c|c|c|c|c|}
\hline \multirow[t]{2}{*}{ Producto } & \multicolumn{4}{|l|}{ Eficiencia } & \multicolumn{4}{|c|}{ Producción } \\
\hline & Unidad & 1961 & 2014 & $\begin{array}{l}\text { Tasa de } \\
\text { cambio }\end{array}$ & Unidad & 1961 & 2014 & $\begin{array}{l}\text { Tasa de } \\
\text { cambio }\end{array}$ \\
\hline Fertilizante $\mathrm{N}^{\mathrm{a}}$ & $\mathrm{t} \mathrm{N} / \mathrm{MJ}$ & 10.3 & 22.2 & $x 2.15$ & Mt & 11 & 103 & x9.32 \\
\hline Carne vacunab & $\mathrm{kg}$ /animal ${ }^{\mathrm{c}}$ & 160 & 215 & x1.34 & Mt & 28 & 64 & $x 2.33$ \\
\hline Carne aviar ${ }^{b}$ & $\mathrm{~kg} /$ animal $^{\mathrm{c}}$ & 1.15 & 1.62 & x1.41 & Mt & 8 & 100 & x13.3 \\
\hline Carne porcina ${ }^{b}$ & kg/animal ${ }^{\mathrm{c}}$ & 66 & 80 & x1.21 & Mt & 25 & 117 & $x 4.73$ \\
\hline Leche vacuna $^{\mathrm{b}}$ & kg/animalc & 1768 & 2401 & x1.35 & Mt & 314 & 655 & $x 2.09$ \\
\hline Huevos $^{\mathrm{b}}$ & kg/animal ${ }^{\mathrm{c}}$ & 7.60 & 9.86 & x1.28 & Mt & 14 & 70 & $\times 4.87$ \\
\hline Maíz ${ }^{b}$ & $\mathrm{t} / \mathrm{ha}$ & 1.94 & 5.59 & $x 2.88$ & Mt & 205 & 1039 & $x 5.06$ \\
\hline Trigo $^{b}$ & t/ha & 1.08 & 3.30 & x3.05 & Mt & 222 & 726 & x3.27 \\
\hline Arroz $^{b}$ & t/ha & 1.86 & 4.52 & $x 2.43$ & Mt & 215 & 742 & x3.45 \\
\hline
\end{tabular}

a Pellegrini and Fernández (2018); ${ }^{b}$ FAOSTAT (2020); c durante toda su vida útil. Ver Material Suplementario.

de los cultivos durante el período 1965-2004 redujo la demanda mundial de tierras agrícolas, en relación a la que hubiese habido por la presión del crecimiento poblacional, en unos 18-27 millones de hectáreas, evitando - entre otras cosas - la deforestación de 2 millones de hectáreas en países en vías de desarrollo (Stevenson et al. 2013). Sin embargo, otras consecuencias negativas derivadas del Efecto Rebote debido al mejoramiento tecnológico con el objetivo de incrementar la productividad de los cultivos han sido resaltadas recientemente (Hamant 2020; Gras and Cáceres 2020).

Si no existiese el Efecto Rebote, el factor de incremento de la producción (última columna de la Tabla 1) debiera haber sido: a) o bien similar al factor de incremento de la eficiencia (equivalente a un uso constante de recursos), o bien b) inferior a ese factor, equivalente al descenso de uso de insumos en respuesta al aumento de eficiencia (como en la hipótesis de land sparing). La Tabla 1 indica que esto no sucedió. Organizaciones como la Agencia Internacional de Energía han ignorado o minimizado el fenómeno durante años, pero durante la última década se han publicado cientos de artículos que refuerzan la idea de que el Efecto Rebote es más pronunciado de lo que se suponía (Berners-Lee 2019; Ruzzenenti et al. 2019).

El caso del sector agropecuario presenta las similitudes resaltadas, pero también diferencias con otros sectores productivos, ya que provee la materia prima de los alimentos que permitieron más que duplicar la población humana durante las últimas cinco décadas. Aunque el crecimiento poblacional global causó gran parte del aumento de la demanda de alimentos y por lo tanto de los recursos utilizados, las preferencias alimentarias también han jugado un papel destacado en el incremento del uso de recursos naturales y del impacto ambiental (Kastner et al. 2012). Por ejemplo, Alexander et al. (2015) estimaron que, si bien la población aumentó 2.27 veces desde 1961 a 2011, el consumo per cápita de alimentos de origen animal se incrementó en 4.12 veces, dominando así el área bajo producción y siendo responsable del $65 \%$ de los cambios en el uso de la tierra durante ese período.

\section{Somos LO QUE COMEMOS}

A escala global, la Revolución Verde aumentó la disponibilidad de alimentos, medida tanto como oferta de calorías como proteínas por persona, causando una reducción en los precios - otra predicción de Borlaug, que en general sí se cumplió (por lo menos, hasta que los cultivos de grano se empezaron a utilizar como biocombustibles) - y facilitando su accesibilidad para la población (Pingali 2012). Como resultado, la disponibilidad promedio de calorías a escala global pasó de 2.196 kcal.persona $^{-1}$.día ${ }^{-1}$ en 1961 a 2.884 kcal.persona ${ }^{-1}$.día ${ }^{-1}$ en 2013 (Roser and Ritchie 2019). Sin embargo, esas calorías se encuentran mal distribuidas, y hoy todavía hay 820 millones de personas con hambre crónica (déficit de calorías) y 2100 millones de personas con sobrepeso u obesidad (FAO 2019).

El incremento en la disponibilidad de calorías fue determinado por un incremento en la producción de los cultivos, los cuales 
no fueron aquellos más beneficiosos para la salud, como las frutas y las verduras, ni mucho menos las legumbres y los cereales integrales (ver Tabla 2), sino un grupo selecto que fueron aprovechados, y luego promovidos, por la industria para transformarlos en otros alimentos. Dichos cultivos fueron: la caña de azúcar, el maíz, el arroz, el trigo, la soja, el girasol, la palma, la cebada, el centeno, la avena, la papa y la mandioca. Los motivos por los cuales se favorecieron estos cultivos fueron porque la mayoría son relativamente fáciles de almacenar y de transportar (Krausmann and Langthaler 2019), y porque sirven para múltiples propósitos, como alimentar a los seres humanos y al ganado, elaborar biocombustibles, producir alcohol y otros (Borras et al. 2016). En este sentido, salvo el arroz, la papa y la mandioca, estos cultivos son transformados en otros antes de llegar al consumidorfinal, yaseaenalimentosfácilmente identificables con la materia prima (como las harinas), en alimentos de origen animal (carnes, leche y huevos), o en componentes de los alimentos ultraprocesados. Estos últimos se caracterizan por una combinación de azúcares, grasas, sal y otros aditivos (como el jarabe de maíz alto en fructosa, la goma xántica o la lecitina de soja), algunas de las cuales facilitan su almacenamiento prolongado y otras que resultan prácticamente irresistibles para el cerebro del Homo sapiens, moldeado evolutivamente por la escasez de ciertos nutrientes (Aguirre 2007; Small and DiFeliceantonio 2019). Dada su compleja composición y nula transparencia del proceso de preparación (Fischer 1993), han sido llamados OCNIs: Objetos Comestibles No Identificados (Fischer 1993); y son considerados - junto a las bebidas azucaradas - como uno de los factores más influyentes e incluso 'vectores' de la actual pandemia de obesidad y ECNT (Monteiro et al. 2019).

La rápida urbanización, la reducción en el hábito de consumir comida casera, el aumento en los ingresos promedio por persona y el incremento en la disponibilidad de alimentos poco sanos, baratos y adictivos (Carolan 2013), empujaron gradualmente a las sociedades hacia dietas hipercalóricas e insalubres conocidas como 'dietas occidentales' (una 'gastroanomia', en contraposición a la gastronomía, que sigue las reglas de la cocina de la cultura propia) (Pollan 2008). Este patrón alimentario globalizado se caracteriza por presentar una gran proporción de granos refinados (harinas), azúcar, sal y grasas (aceites) agregadas, una importante cantidad de alimentos de origen animal (muchas veces también ultraprocesados), y además una reducción paulatina de las legumbres, verduras y cereales integrales en casi todo el mundo (Popkin 2017; IFPRI 2017). Si bien esta tendencia se observa en toda la población, los más fuertemente afectados son los sectores más empobrecidos (como suele suceder en muchos otros aspectos de la desigualdad) (Bauman 2011; Fernández 2015), lo que ha llevado a tener por primera vez en la historia 'gordos pobres y flacos ricos', como lo destaca Patricia Aguirre (2017).

Las dietas de tipo occidental, ricas en alimentos de origen animal, granos refinados y alimentos ultraprocesados, y pobres en frutas, verduras, granos integrales (tanto cereales como legumbres), frutos secos y semillas, han sido asociadas de forma consistente con un aumento en el riesgo de padecer diversas ECNT como infarto de corazón, accidente cerebrovascular, hipertensión arterial, diabetes tipo 2, obesidad y varios tipos de cáncer (Fanzo et al. 2018). Incluso, se las ha relacionado con enfermedades que poco parecen tener que ver con la alimentación como el Alzheimer, ciertas enfermedades pulmonares (asma y EPOC) y patologías autoinmunes (Willett 2013). De hecho, se estima que a escala global las dietas subóptimas enferman y matan anualmente a más personas que cualquier otro factor de riesgo, incluso más que el tabaquismo, la violencia y los accidentes viales (Afshin et al. 2019). Esto determina que las dietas insalubres - casi siempre combinado con el sedentarismo - tengan un enorme impacto negativo sobre la salud pública, la calidad de vida de las personas, la productividad laboral y los costos sanitarios (Popkin et al. 2012). En líneas generales, se considera que los factores de riesgo dietarios más importantes para el desarrollo de ECNT son el bajo consumo de frutas, verduras, legumbres, cereales integrales, frutos secos y semillas, y el elevado consumo de carnes rojas y procesadas, bebidas azucaradas y alcohólicas, sal y alimentos ultraprocesados (Fardet and Boirie 2014). Tal como lo sugiere un conocido divulgador del tema, "Eat food [evitando OCNIs], not too much, mostly plants" (Pollan 2008).

La importancia de la alimentación para mantener y restablecer la salud fue ampliamente subestimada por la comunidad médica, pero en las últimas décadas se destacó a la alimentación saludable como una de las herramientas más poderosas para promover el bienestar y mejorar la salud 
Tabla 2. Consumo recomendado de doce grupos alimentarios de acuerdo a la Escuela de Salud Pública de Harvard y efecto de su consumo sobre el riesgo de ECNT (enfermedades crónicas no-transmisibles). Estas recomendaciones no implican necesariamente que se deban consumir todos los alimentos en el mismo día.

Table 2. Recommended intake of twelve food groups according to the Harvard School of Public Health guidelines, and its associated risk with the most prevalent NCDs (chronic non-communicable diseases). These recommendations does not necessarily imply that all foods should be consumed on the same day.

\begin{tabular}{|c|c|c|c|c|c|c|c|c|}
\hline Grupo alimentario & $\begin{array}{l}\text { Recomendado } \\
(\text { g/día })^{c}\end{array}$ & $\mathrm{TCM}^{\mathrm{d}}$ & $\mathrm{EC}^{\mathrm{e}}$ & $\mathrm{ACV}^{\mathrm{e}}$ & $\mathrm{IAM}^{\mathrm{e}}$ & $\mathrm{DBT}^{\mathrm{f}}$ & $\mathrm{HTA}^{\mathrm{g}}$ & $\mathrm{CC}^{\mathrm{h}}$ \\
\hline Verduras & 300 & $\downarrow$ & $\downarrow$ & $\downarrow$ & $\downarrow$ & $\downarrow$ & $\leftrightarrow$ & $\downarrow$ \\
\hline Verduras ricas en almidón ${ }^{\mathrm{i}}$ & 50 & $\leftrightarrow$ & $\leftrightarrow$ & $\leftrightarrow$ & $\leftrightarrow$ & $\uparrow$ & $\leftrightarrow$ & $\leftrightarrow$ \\
\hline Frutas & 200 & $\downarrow$ & $\downarrow$ & $\downarrow$ & $\leftrightarrow$ & $\downarrow$ & $\downarrow$ & $\downarrow$ \\
\hline Frutos secos & 25 & $\downarrow$ & $\downarrow$ & $\leftrightarrow$ & $\leftrightarrow$ & $\leftrightarrow$ & $\downarrow$ & $\leftrightarrow$ \\
\hline Legumbres & 100 & $\downarrow$ & $\downarrow$ & $\leftrightarrow$ & NE & $\leftrightarrow$ & $\leftrightarrow$ & $\leftrightarrow$ \\
\hline Cereales integrales $^{\mathrm{a}}$ & 232 & $\downarrow$ & $\downarrow$ & $\leftrightarrow$ & $\downarrow$ & $\downarrow$ & $\downarrow$ & $\downarrow$ \\
\hline Cereales refinados & 0 & $\leftrightarrow$ & $\leftrightarrow$ & $\leftrightarrow$ & $\leftrightarrow$ & $\leftrightarrow$ & $\leftrightarrow$ & $\mathrm{NE}$ \\
\hline Huevos & 13 & $\uparrow$ & $\leftrightarrow$ & $\leftrightarrow$ & $\uparrow$ & $\leftrightarrow$ & $\downarrow$ & $\leftrightarrow$ \\
\hline Lácteos & 250 & $\leftrightarrow$ & $\leftrightarrow$ & $\downarrow$ & $\uparrow$ & $\downarrow$ & $\downarrow$ & $\downarrow$ \\
\hline Pescado & 28 & $\downarrow$ & $\downarrow$ & $\downarrow$ & $\downarrow$ & $\leftrightarrow$ & $\leftrightarrow$ & $\downarrow$ \\
\hline Carnes rojas ${ }^{\mathrm{b}}$ & 14 & $\uparrow$ & $\uparrow$ & $\uparrow$ & $\uparrow$ & $\uparrow$ & $\uparrow$ & $\uparrow$ \\
\hline Carnes procesadas & 0 & $\uparrow$ & $\uparrow$ & $\uparrow$ & $\uparrow$ & $\uparrow$ & $\uparrow$ & $\uparrow$ \\
\hline Gaseosas & 0 & $\uparrow$ & $\uparrow$ & $\uparrow$ & $\uparrow$ & $\uparrow$ & $\uparrow$ & $\leftrightarrow$ \\
\hline
\end{tabular}

TCM: todas las causas de muerte; EC: enfermedad coronaria; ACV: accidente cerebrovascular; IAM: infarto agudo TCM: todas las causas de muerte, EC: enfermedad coronaria; ACV: accidente cerebrovascular; de miocardio; DBT2: diabetes mellitus tipo 2; HTA: hipertensión arterial; CC: cáncer de colon. $\leftrightarrow:$ no hay asociación
de entre consumo y riesgo; $\uparrow$ : asociación positiva entre consumo y riesgo; $\downarrow$ : asociación negativa entre consumo y riesgo; NE: no establecido.

a Corresponde al peso cocido de los cereales. ${ }^{\mathrm{b}}$ Carne vacuna. porcina y caprina; para la carne aviar aún no existe información de calidad comparable. c Wang et al. (2019). d Schwingshackl et al. (2017a). " Bechthold et al. (2019). ${ }^{\mathrm{f}}$ Schwingshackl et al. (2017b). ${ }^{\mathrm{g}}$ Schwingshackl et al. (2017c). h Schwingshackl et al. (2018). ${ }^{\mathrm{i}}$ Schwingshackl et al. (2019).

pública (Li et al. 2020). Hasta hace algunas décadas, el foco de la ciencia de la nutrición humana estaba en prevenir deficiencias en la dieta y alcanzar la ingesta recomendada de algunos nutrientes esenciales (con énfasis en las calorías y proteínas), dando lugar a las recomendaciones basadas en los Cuatro Grupos Alimentarios Básicos de una dieta saludable: 1) carnes, 2) lácteos, 3) granos, y 4) frutas y verduras (Willet and Stampfer 2013). Posteriormente, los estudios epidemiológicos y los ensayos clínicos aleatorizados arrojaron luz sobre cómo los factores de riesgo dietarios afectan la salud a largo plazo, indicando que la reducción o eliminación de los alimentos perjudiciales y el aumento de la ingesta de los alimentos protectores (Tabla 2) son capaces de prevenir la mayoría de los casos de las ECNT, así como de reducir las muertes prematuras, demostrando que dichas enfermedades no son consecuencias inevitables de la sociedad moderna sino de la inactividad física, el tabaquismo, el consumo excesivo de alcohol y las dietas insalubres (Bechthold et al. 2018). En un estudio reciente se estimó que el mejoramiento en la calidad de la dieta podría ser capaz de prevenir el $24 \%$ de las muertes prematuras en el mundo (para la Argentina se estimó un 29\%): 3.9 millones de muertes por enfermedades coronarias, 1.6 (en las mismas unidades) por cáncer, 1.7 por enfermedades respiratorias, 1.2 por enfermedades digestivas, 1 por accidentes cerebrovasculares, 0.6 por diabetes tipo 2, 0.5 por enfermedades renales y 0.4 por enfermedades neurodegenerativas (Wang et al. 2019). El impacto del consumo de alimentos sobre la salud pública se vio resaltado durante la pandemia de COVID19 , ya que las personas más afectadas por las formas graves de la enfermedad fueron aquellas que presentaban una o más ECNT (Clark et al. 2020; CDC 2020; IATF 2020). De esta manera, comer de manera saludable es más importante que nunca (Godlee 2020).

En el contexto de la relación entre dietas y salud discutidos en los párrafos anteriores, la definición de 'dieta saludable' evolucionó para enfocarse en la optimización de la salud a largo plazo, considerando tanto los problemas de salud por deficiencias como también por excesos (Céspedes and Hu 2015). Los expertos en salud propusieron cambiar la antigua visión centrada en la ingesta de nutrientes - que Carolan (2013) llama la ideología reduccionista del 'nutricionismo' - hacia 


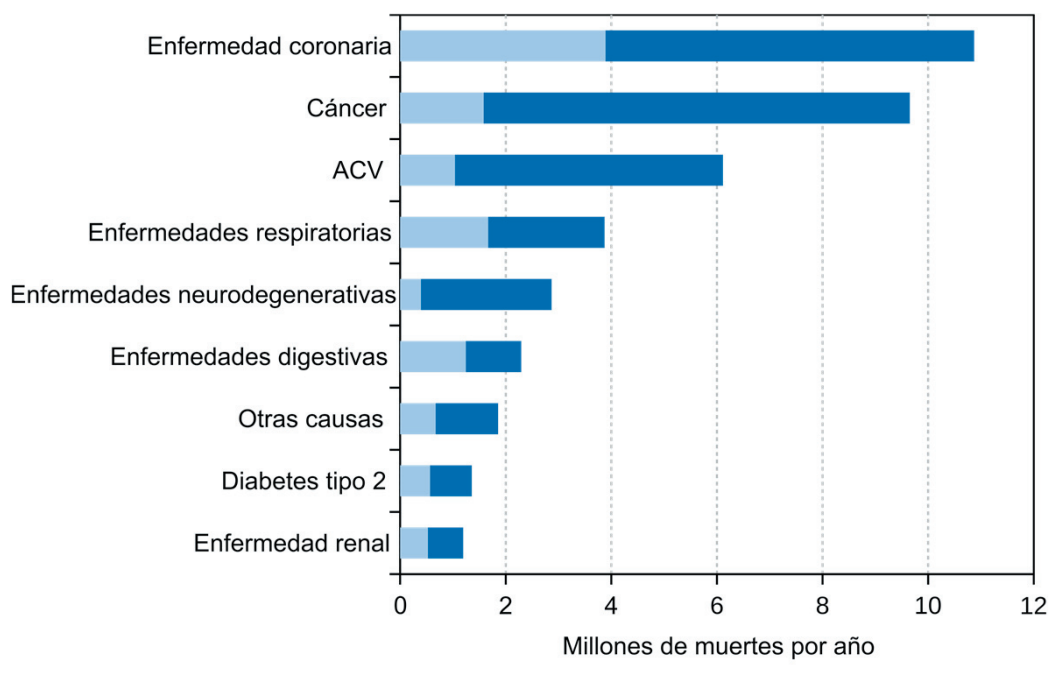

Figura 1. Muertes prematuras prevenibles en el mundo por enfermedades crónicas notransmisibles (ECNT) como resultado de la adopción de dietas saludables (en celeste) en relación con las muertes prematuras totales (en azul). Basada en Wang et al. (2019).

Figure 1. Preventable premature deaths worldwide due to Chronic Noncommunicable Diseases (NCDs) as a result of the adoption of healthy diets (in light blue) in relation to total premature deaths (in blue). Based on Wang et al. (2019). otra enfocada en incentivar el consumo de grupos alimentarios protectores de la salud y limitar la ingesta de grupos de alimentos insalubres (Tabla 2), contemplando al mismo tiempo los hábitos dietarios de las diferentes poblaciones y las enfermedades prevalentes de cada región (Albert 2007; Céspedes et al. 2015). Poco a poco, el fomento de dietas saludables se convirtió en una política de estado de muchos países (FAO 2020). En 1992, la Organización Mundial para la Alimentación y la Agricultura (FAO) y la Organización Mundial de la Salud (OMS) sugirieron que cada país debería desarrollar sus propias recomendaciones dietarias a fines de orientar a la población y los profesionales de salud (FAO/WHO 1992), y así nacieron las Guías Alimentarias Basadas en Alimentos (GABA). En la Argentina, después de un esfuerzo colectivo y coordinado a nivel nacional entre miembros de la Asociación Argentina de Dietistas y Nutricionistas, se publicó hace casi dos décadas la primera Guía Alimentaria para la Población Argentina (GAPA) con el objetivo de "[...] combatir hábitos alimentarios perjudiciales y reforzar aquellos adecuados para mantener la salud" (Silva 2003). Debido a que el perfil nutricional de los argentinos se acerca más al de los países de altos ingresos (dietas occidentales) que al de los países de bajos-medianos ingresos, la última actualización recomienda reducir el consumo de carnes, alimentos ultraprocesados y gaseosas, y de incrementar el consumo de frutas, verduras, frutos secos y legumbres (Ministerio de Salud 2016).

Las dietas vegetarianas y veganas, vistas en el pasado con sospechas como una rareza, son actualmente relativamente populares y aceptadas, y están en expansión en muchos países por ser consideradas alternativas saludables (y por motivos filosóficos y ambientales). Aunque es frecuente escuchar que la eliminación de las carnes y otros alimentos de origen animal puede generar problemas a la salud, el consenso científico muestra lo contrario. Diversas instituciones científicas (como la Academia de Nutrición y Dietética de los Estados Unidos y la Sociedad Argentina de Nutrición) encontraron evidencias suficientes para afirmar que las dietas vegetarianas bien planificadas —incluso las estrictas que no contienen carnes, huevos ni lácteos - son saludables, nutricionalmente adecuadas y apropiadas para cualquier etapa de la vida, incluyendo embarazo, lactancia, infancia, niñez, adolescencia, adultez y vejez e incluso para atletas (Gallo et al. 2015; Melina et al. 2016; Arrieta 2017). Sin embargo, es importante resaltar la importancia que tiene la planificación adecuada de las dietas con el asesoramiento de un profesional de la nutrición - recomendación extendida a toda la población debido a la baja calidad dietaria general - principalmente para evitar las posibles deficiencias nutricionales, especialmente de vitamina $B_{12}$ (cuyo déficit puede causar anemia y trastornos neurológicos [Aguirre et al. 2019]). La ingesta de un suplemento de vitamina $B_{12}$ es suficiente para asegurar la ingesta adecuada de este vital nutriente. A pesar de que la presentación suele asemejarse a un medicamento (como pastilla o polvo efervescente), no hay riesgos asociados cuando se la ingiere en las dosis recomendadas, y además su producción es muy barata y sustentable debido a que se obtiene a partir de la fermentación por parte de algunas bacterias modificadas genéticamente (Fang et al. 2017). 


\section{Win-WIN: SALUd HUMANA Y AMBIENTAL}

Diversos estudios publicados en la última década identificaron que la adopción de dietas saludables no sólo estaría relacionada con una reducción del riesgo de desarrollar ECNT sino también (en general) con un menor impacto ambiental, con reducciones en el consumo de energía fósil, emisión de GEI, demanda de tierras agrícolas, uso de agua dulce y eutrofización, entre otros (Willet et al. 2019; Vermeulen et al. 2019; Leclere et al. 2020). Esto en parte ocurre porque, debido a un principio ecológico básico, se necesita menos energía solar (y por lo tanto menos área total) para producir una caloría de alimentos de origen vegetal que una de origen animal. Así, en general los segundos demandan más recursos que los primeros para ser producidos (e.g., tierra, energía, nitrógeno, fósforo, agua, etc.) y emiten más contaminantes $\left(\mathrm{CO}_{2^{\prime}} \mathrm{CH}_{4^{\prime}}\right.$ $\mathrm{NO}_{x^{\prime}} \mathrm{SO}_{4}^{-2}$ y $\left.\mathrm{PO}_{3}^{-3}\right)$, tanto si se los compara por unidad de peso como de nutrientes (con diferencias de hasta 20 veces) (González et al. 2011; Clark and Tilman 2017; Poore and Nemecek 2018). En otras palabras, hay una relación aparentemente virtuosa entre la capacidad protectora para la salud que tiene cada grupo de alimentos y el bajo impacto ambiental asociado a su producción (Figura 2). Este hallazgo se refuerza porque, en líneas generales, aquellos alimentos que reducen el riesgo de una ECNT también lo hacen con las otras ECNT (Tabla 2), y los que presentan valores más bajos en un indicador de impacto ambiental para su producción también lo hacen en otros indicadores (Clark et al. 2019). Esto significa que la propuesta de incrementar la proporción de frutas, verduras y granos integrales, y de reducir la participación de algunos alimentos de origen animal (carnes rojas y carnes procesadas), le otorgaría a las GABA el título de dietas tanto saludables como sostenibles (Fischer and Garnett 2016; Behrens et al. 2017). Aunque actualmente está en discusión de si las GABA son realmente sustentables a pesar de ser saludables (Springmann et al. 2020).

Las correlaciones positivas dentro y entre los ejes de la Figura 2 permiten una estrategia comunicacional muy simple y contundente, lo que hasta cierto punto es ventajoso por lo

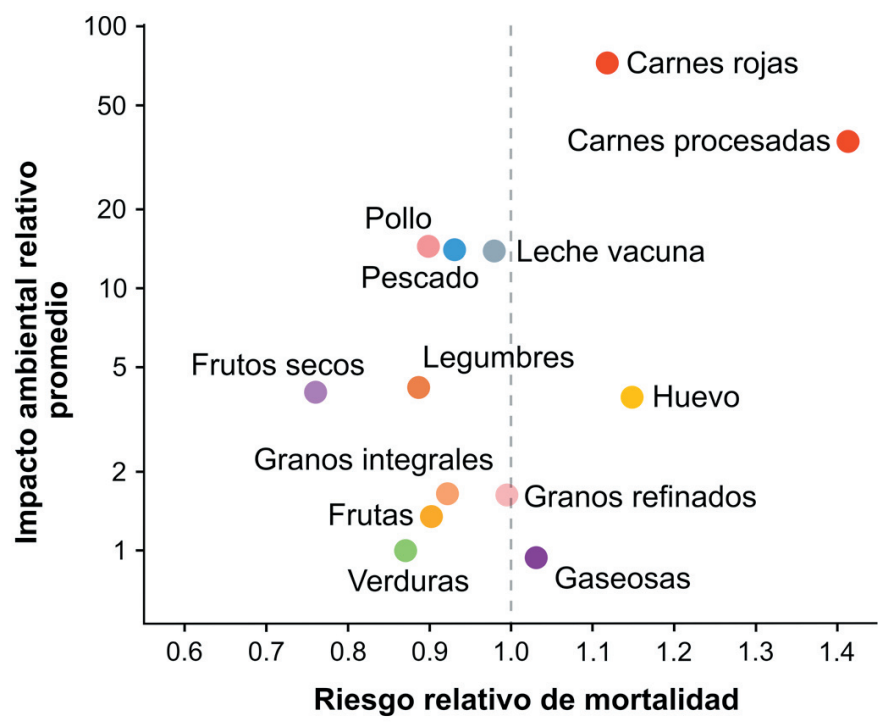

Figura 2. Asociación entre el riesgo relativo de mortalidad y el impacto ambiental relativo promedio de 12 grupos de alimentos (datos de Clark et al. 2019; en su mayoría para países desarrollados y áreas húmedas). Cuando el riesgo relativo de mortalidad es $>1$, el consumo de una porción adicional del grupo alimentario está asociado a un incremento en el riesgo de mortalidad por todas las causas; cuando es <1, existe una reducción del riesgo. El impacto ambiental relativo promedio expresa el promedio de cinco indicadores de impacto ambiental por $\mathrm{kg}$ de producto (consumo de energía fósil, emisiones de GEI, uso de la tierra, potencial de eutrofización y potencial de acidificación), relativizado respecto al impacto de $1 \mathrm{~kg}$ de verduras.

Figure 2. Relationship between relative risk on mortality and averaged relative environmental impact of twelve food groups (data from Clark et al. 2019; mostly for developed countries and humid areas). When the relative risk of mortality is $>1$, the consumption of an additional daily serving of the food group is associated with an increase in the risk of mortality from all causes; a relative risk $<1$ indicates that this consumption is associated with lowered mortality risk. The averaged relative environmental impact expresses the average of five environmental impact indicators per kilogram of weight (fossil energy consumption, GHG emissions, land use, eutrophication potential and acidification potential), relativized with respect to the impact of $1 \mathrm{~kg}$ of vegetables. 
didáctico, pero que corre los riesgos de toda sobresimplificación. Por ejemplo, una cuestión muy discutida en relación a la producción de carne vacuna es la emisión de GEI (Jaurena 2019). Si consideramos sólo las emisiones de metano (importante GEI) calculadas a partir de las guías del Panel Intergubernamental para el Cambio Climático (IPCC 2019), podríamos deducir que la ganadería bovina alimentada a base de granos tiene menor impacto ambiental que la más extensiva con base pastoril, ya que los animales alimentados con pasto tienen una mayor emisión de metano que los animales alimentados con granos (De Vries et al. 2015). Sin embargo, existen otros importantes impactos de la producción bovina intensiva que esta métrica centrada en los animales individuales no muestra, como las emisiones de dióxido de carbono y de óxido nitroso (ambos importantes GEIs) derivados de la producción de los granos utilizados como suplemento (Tilman et al. 2011) - que en la Tabla 1 se listan como productos, pero en este tipo de ganadería pasan a ser insumos, la erosión y pérdidas de otros servicios ecosistémicos causada por los cultivos anuales por sobre las ventajas de las pasturas perennes y los pastizales naturales, así como el uso de antibióticos y el desarrollo de resistencia a ellos, entre otros (Picasso et al. 2014; Garibaldi et al. 2018). Además, alimentar al ganado bovino con granos es, como se dijo anteriormente, ineficiente desde el punto de vista del uso de las tierras con capacidad agrícola, porque en este caso los animales compiten por alimentos que podrían ser consumidos directamente por los humanos (van Zanten et al. 2018). Esto último es de una importancia crucial, ya que producir esos granos tiene sus propio impacto ambiental asociado. De hecho, se estima que a escala global la ganadería de rumiantes - principalmente vacunos, incluyendo su producción láctea - usa directa y, sobre todo, indirectamente, tanta tierra de cultivo, agua y fertilizantes como la suma de los sectores porcinos y aviar (46\% entre rumiantes y estos monogástricos) (Herrero et al. 2015). En la Argentina, a pesar de que el sector bovino para carne es fundamentalmente pastoril - $92 \%$ de la biomasa total consumida por el sector es pasto - , en el año 2016 consumió más maíz que el sector porcino y aviar juntos (6.25 vs. 5.66 millones de toneladas) (Arrieta et al. 2020; Arrieta et al. en revisión). Otro nivel de complejidad para agregar al análisis es si la ganadería se realiza sobre pastizales y pasturas existentes o sobre áreas en las que se eliminó la vegetación leñosa (e.g., arbustales o bosques nativos), en cuyo caso las emisiones de dióxido de carbono debidas al cambio en el uso de la tierra y el impacto sobre la biodiversidad serán apreciables (Persson et al. 2014). Esto es un ejemplo de la necesidad de realizar análisis integrales tomando múltiples indicadores, no solo a nivel del producto (por kg) o del sistema productivo, sino también a escala del sistema agroalimentario (Notarnicola et al. 2017).

A pesar del mayor impacto ambiental de los alimentos de origen animal respecto a los de origen vegetal, la eliminación total de las carnes — si bien médicamente posible, como se explicó - resulta innecesaria desde la perspectiva de la sustentabilidad (Röös et al. 2015; Adesogan et al. 2020). Esto se aplica particularmente a los casos en los que la carne se produce en sistemas pastoriles con bajo uso de insumos (tanto aves como cerdos y vacunos) o si el forraje no compite con la provisión de alimentos a los humanos, tal como sucede en zonas áridas a subhúmedas o con suelos poco aptos (van Zanten et al. 2018). Además, en los sistemas productivos actuales el ganado tiene dos ventajas (Herrero et al. 2010): a) los rumiantes transforman en proteínas la fibra de baja calidad la oferta forrajera de las tierras sin aptitud agrícola, y b) permiten el restablecimiento de la fertilidad físico-química de los suelos a través de las rotaciones agrícolo-ganaderas en los suelos de mayor calidad (manejo tradicional que se ha ido abandonando y debería restablecerse). Aunque en muchos casos los animales no son consumidos por los productores y sus familias, sino que son vendidos en el mercado local para comprar otros alimentos, la tenencia de animales puede representar una importante fuente de energía, proteínas y otros valiosos nutrientes en momentos de precios muy bajos o para poblaciones de bajos recursos que viven en zonas marginales y no acceden a los mercados (Smith et al. 2013; Adesogan et al. 2020). De todos modos, en general no son estas comunidades las que presentan los mayores consumos de alimentos de origen animal, sino los habitantes de las zonas urbanas, particularmente aquellos con ingresos medios y altos (Godfray et al. 2018).

En definitiva, los alimentos que elegimos para comer tienen un impacto tanto en nuestra salud como en el ambiente, y la promoción de las dietas saludables representa una gran oportunidad para generar beneficios en ambas esferas (situación win-win). 
Los argentinos, ¿Pueden adoptar UNA DIETA SALUDABLE Y SOSTENIBLE?

Si bien es cierto que los cultivos extensivos destinados a la exportación representan un importante factor de degradación ambiental en la Argentina (Viglizzo et al. 2011), la participación del mercado interno en el impacto ambiental del sistema alimentario nacional es considerable. En el 2017 la producción de alimentos destinados al mercado interno requirió de unas 60 Mha de superficie agropecuaria (de las cuales 8 Mha correspondían a tierras de cultivo), 106 terajoules (TJ) de energía fósil y $2.3 \mathrm{~km}^{3}$ de agua dulce, mientras que emitió $99 \mathrm{Mt} \mathrm{CO}_{2}$-eq y $0.24 \mathrm{Mt} \mathrm{PO}_{4}$-eq (Arrieta et al. en revisión).

El sentido común puede hacernos creer que, por el hecho de vivir en un país autodenominado históricamente el 'granero' o el 'supermercado' del mundo, la adopción de una dieta saludable (y sostenible) es meramente una cuestión de voluntad y educación. Sin embargo, la afirmación de que la Argentina es capaz de alimentar a 400 millones de personas está basada en un cálculo simplista que contempla sólo la cantidad de calorías producidas y su demanda por persona (Slipczuk 2019). Si bien cubrir las necesidades energéticas de la población nos permite combatir hasta cierto punto el hambre, no es suficiente para llevar una vida activa y sana. Para adoptar una dieta saludable las personas necesitamos no sólo de la información y la voluntad, sino también del acceso físico y económico a suficientes alimentos inocuos, nutritivos y culturalmente aceptables; es decir, de seguridad alimentaria (Gordillo and Jerónimo 2013). Tal como se muestra en la Tabla 3, el patrón de consumo de alimentos en la Argentina se ve condicionado por la limitada disponibilidad de los alimentos en los comercios.

Tabla 3. Flujo de masa de catorce grupos alimentarios a lo largo de la cadena agroalimentaria en la Argentina. Los valores están expresados en gramos por persona y por día para el año 2017. Los datos presentados aquí no representan el consumo real, sino la disponibilidad de alimentos en los comercios. Más detalles en Material Suplementario.

Table 3. Mass flow of fourteen food groups throughout the food chain in Argentina. The values are expressed in grams per person and per day for the year 2017. The data presented here does not represent real consumption, but rather the food availability. Details in Supplementary Material.

\begin{tabular}{|c|c|c|c|c|c|c|c|c|}
\hline 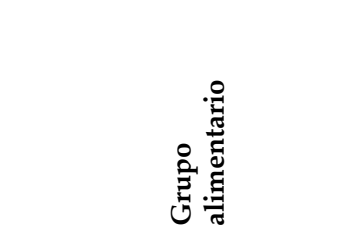 & 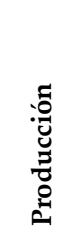 & 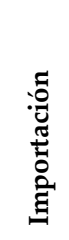 & 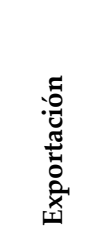 & 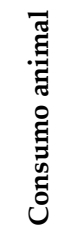 & 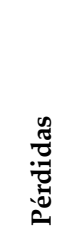 & 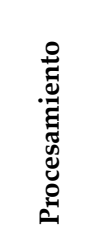 & 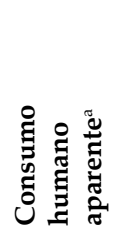 & 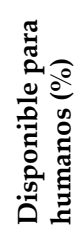 \\
\hline Verduras & 210 & 7.23 & 9.29 & 0 & 22.76 & 0 & 185 & 88 \\
\hline Verduras ricas en almidón & 186 & 3.87 & 23.82 & 5.86 & 17.46 & 19.52 & 110 & 58 \\
\hline Frutas & 472 & 43 & 118.41 & 0 & 37.23 & 119.91 & 257 & 54 \\
\hline Frutos secos & 65 & 0.37 & 46.52 & 0 & 0.69 & 16.77 & 0.87 & 1 \\
\hline Legumbres $^{b}$ & 44 & 0.12 & 39.35 & 0.06 & 0.81 & 0 & 2.24 & 5 \\
\hline Oleaginosas ${ }^{\mathrm{c}}$ & 3691 & 118 & 475 & 293 & 42 & 2888 & 0 & 0 \\
\hline Cereales & 4771 & 3 & 2631 & 1017 & 79 & 66 & 358 & 7 \\
\hline Huevos & 51 & 0 & 0.5 & 0 & 2.56 & 0 & 42 & 84 \\
\hline Lácteos & 632 & 3.37 & 90.48 & 38.1 & 5.05 & 0 & 438 & 70 \\
\hline Carne vacuna $^{\mathrm{d}}$ & 177 & 0.06 & 20.95 & 0 & 8.85 & 0 & 148 & 84 \\
\hline Carne caprina $^{\mathrm{d}}$ & 4 & 0 & 0.12 & 0 & 0.19 & 0 & 3.49 & 90 \\
\hline Carne porcina $^{\mathrm{d}}$ & 35 & 2.18 & 0.19 & 0 & 1.75 & 0 & 29 & 83 \\
\hline Carne aviar & 135 & 0.5 & 12.91 & 0 & 6.61 & 0 & 116 & 86 \\
\hline Carne de pescado & 28 & 4 & 19 & 0 & 0 & 0 & 12 & 44 \\
\hline Carnes procesadas & NC & $\mathrm{NC}$ & NC & $\mathrm{NC}$ & $\mathrm{NC}$ & NC & 21.5 & 100 \\
\hline Ultraprocesados & NC & $\mathrm{NC}$ & NC & $\mathrm{NC}$ & $\mathrm{NC}$ & $\mathrm{NC}$ & 81 & 100 \\
\hline Bebidas azucaradas ${ }^{\mathrm{e}, \mathrm{f}}$ & NC & $\mathrm{NC}$ & $\mathrm{NC}$ & $\mathrm{NC}$ & $\mathrm{NC}$ & NC & 206 & 100 \\
\hline
\end{tabular}

NC: no corresponde.

${ }^{a}$ Consumo humano aparente (alimento disponible para humanos) $=$ stocks iniciales + (importación + producción)

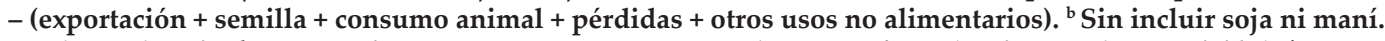

${ }^{\mathrm{c}}$ Incluyendo soja. ${ }^{\mathrm{d}}$ Carnes rojas. ${ }^{\mathrm{e}}$ Consumo aparente para el año 2017/2018 (Arrieta et al. en revisión). ${ }^{\mathrm{f}}$ En $\mathrm{ml} /$ día. 


\section{Alimentos que consumimos poco}

Frutas y verduras. Durante el año 2017 la producción nacional de frutas y verduras alcanzó los $659 \mathrm{~g} /$ día por persona. Sin embargo, la disponibilidad al punto de venta fue de 367 g/día, 70\% mayor a los datos registrados de compras de frutas y verduras (Arrieta et al. en revisión), aunque por debajo de los valores de consumo recomendados (500 g/día) (Tabla 2). Esta tendencia de bajo consumo coincide con los resultados de la Cuarta Encuesta Nacional de Factores de Riesgo, donde se encontró que sólo $6 \%$ de la población argentina cubrió la recomendación mínima de $400 \mathrm{~g} /$ día de frutas o verduras frescas (MSyDS 2019). Esta realidad no es un fenómeno distintivo de nuestro país, sino que representa la norma en casi todo el mundo (Mason-D'Croz et al. 2019). En el caso de las frutas, el $25 \%$ de la producción fue importada, otro $25 \%$ fue procesada por la industria y sólo el $55 \%$ de la producción total llegó al punto de venta (gracias a un 5\% residual del año anterior). Para las verduras, la situación fue otra, ya que $88 \%$ de la producción llegó al punto de venta, siendo las pérdidas durante la cadena agroalimentaria el mayor contribuyente a la brecha (11\%).

Frutos secos. En el año 2017 se produjeron unos $65 \mathrm{~g} /$ día por persona (con cáscara incluida). Este número contrasta enormemente con los $0.87 \mathrm{~g} /$ día disponibles en los comercios, casi unas 30 veces menos de lo recomendado y cuyo valor puede ser aun menor en lo que respecta a consumo real (más de la mitad del peso de la nuez corresponde a la cáscara). La exportación y el procesamiento industrial explican prácticamente toda la brecha entre producción y consumo $(71 \%$ y $26 \%$, respectivamente).

Legumbres. Sin tener en cuenta la soja, la producción nacional alcanzó los $44 \mathrm{~g} /$ día por persona en el 2017. A este cálculo debemos sumar al maní, ya que a pesar de que se lo suele considerar un fruto seco se trata en realidad de una legumbre: para el año 2017 se produjo 1 millón de toneladas, lo que agrega al grupo unos $62 \mathrm{~g}$ /día por persona. Desafortunadamente, sólo el $5 \%$ de la producción total de legumbres se consume dentro del país, dejando una disponibilidad al mercado interno de $2 \mathrm{~g}$ /día (50 veces menos que lo recomendado).

Carne de pescado. La producción alcanzó los $28 \mathrm{~g}$ /día por persona en el año 2017, valor similar al recomendado. Sin embargo, el 70\% fue exportado, aunque unas 62 mil toneladas importadas contribuyeron a la disponibilidad de ese alimento en el país, por lo que el consumo aparente fue casi menos de la mitad de lo recomendado.

\section{Alimentos que consumimos en exceso}

Verduras ricas en almidón. El consumo aparente de papa, batata y mandioca en la Argentina en 2017 fue de 110 g/día, un poco más de la mitad de lo producido y el doble de lo recomendado. A pesar de la tradicional presencia de estos alimentos en las cocinas de muchas sociedades antiguas y modernas, las recomendaciones actuales sugieren consumirlos con moderación debido a su elevado índice glucémico (una medida de la rapidez con la que un alimento puede elevar el nivel de glucosa en la sangre) y su relación con el desarrollo de diabetes tipo 2 (Schwingshackl et al. 2019).

Carnes rojas y procesadas. En el 2017, 83\% de la carne roja producida en el país fue destinada al mercado interno, contabilizando un consumo aparente total de $181 \mathrm{~g} /$ día por persona (13 veces más de lo recomendado). Sin embargo, estos números pueden ser algo engañosos porque FAOSTAT no reporta la carne lista para consumo, sino como carcasa (la misma pierde $30 \%$ de la masa antes de llegar al consumidor, y otro tanto más durante la cocción) (Arrieta and González 2019). Además, la mitad de la carne porcina es transformada - junto a otras carnes - en carnes procesadas como chorizos, fiambres y embutidos. El mejor dato disponible refiere que el consumo promedio de carnes rojas y carnes procesadas en la Argentina es de 120 g/día y 19 g/día, respectivamente (Arrieta et al. en revisión). Si bien existen diferencias entre estratos socio-económicos, el consumo promedio en el sector más pobres es más alto que el promedio mundial: $63 \mathrm{~g} /$ día de carnes rojas y $8 \mathrm{~g} /$ día de carnes procesadas (mientras que en el sector más rico es de 183 g/día y 32 g/día) (Arrieta et al. en revisión). Así, el consumo de carnes rojas y carnes procesadas se encuentra muy por encima de lo recomendado para la prevención de ECNT, incluso en los estratos sociales de bajos recursos. Las carnes rojas y procesadas han sido vinculadas, de manera consistente y en múltiples estudios, con un mayor riesgo de enfermedades cardiovasculares, diabetes tipo 2 , cáncer y muerte prematura (Larson and Orsini 2014; Wang et al. 2016; WCRF/AICR 2018; Qian et al. 2020). La evidencia sugiere que dicha asociación se debe a la acción de diversas sustancias que se encuentran en 
el tejido animal, que se agregan durante el procesamiento de las carnes o que se producen durante el proceso digestivo en los intestinos. Estas sustancias pueden actuar como carcinógenos (nitratos, nitritos y nitrosaminas [Santarelli et al. 2008; Bouvard et al. 2015]), como inductores de hipertensión arterial (sal [Micha et al. 2010]) y de arteriosclerosis (L-carnitina, grasas saturadas y grasas trans [Siri-Tarino et al. 2010; Koeth et al. 2012]), y como diabetógenos al alterar la función del páncreas (hierro hemo y leucina [Zoncu et al. 2011 Shah and Fonseca 2011; Feskens et al. 2013]). Las toxinas ambientales acumuladas en la grasa también pueden alterar la función pancreática e inducir diabetes tipo 2 (Magliano et al. 2014).

Alimentos ultraprocesados (incluye bebidas azucaradas). En el 2017/2018 el consumo promedio de ultraprocesados representó el $15 \%$ de las calorías totales, siendo las bebidas azucaradas las mayores responsables (Arrieta et al. en revisión). Sin embargo, debido a las limitaciones de la fuente de datos utilizada en el análisis, es posible que el consumo de ultraprocesados sea aún mayor (PAHO 2019).

\section{Desafíos de adoptar una dieta saludable y sostenible}

De lo anterior surge que el primer obstáculo para la adopción de una dieta saludable en la Argentina es la baja disponibilidad de frutas, verduras, frutos secos y pescado. Un camino posible para solucionar dicha escasez podría ser la generación de incentivos a medianos y grandes productores dado que ya disponen del capital para abastecer dicha demanda. Pero debido a que la mayor proporción de estos alimentos es producida por la agricultura familiar y campesina (excepto el pescado), incrementar la disponibilidad de los alimentos saludables brinda también una oportunidad para el desarrollo del sector mediante la formulación e implementación de políticas públicas como la Ley de Reparación Histórica de la Agricultura Familiar (Ley № 27118) y la Ley de Presupuestos Mínimos de Protección y Fortalecimiento de los Territorios Periurbanos Productivos (presentada en la Honorable Cámara de Diputados de la Nación a fines de junio de 2020). Sin embargo, surgen algunas preguntas al pensar en un incremento de la producción de estos alimentos: ¿Cómo afrontaremos el aumento de la demanda de tierras para la producción frutihortícola? ¿Se fomentará la creación de cinturones frutihortícolas periurbanos o la producción se mantendrá en las zonas ecológicamente más aptas? ¿Apostaremos al desarrollo de la producción agroecológica o mantendremos los sistemas productivos tal cual están en el presente? ¿Dedicaremos las reservas de agua subterránea (finitas) a la producción de frutos secos? ¿Se habilitarán más permisos de pesca o se fomentará la acuicultura? ¿Se regulará la acuicultura para evitar la contaminación de los cuerpos de agua? ¿Cuál será la política de precios y otras que aseguren una oferta interna accesible en relación con las demandas del sector exportador? ¿Cuál será la respuesta de la sociedad?

La disponibilidad de alimentos en los comercios es un requisito fundamental para su consumo, pero no es condición suficiente para que se concrete. Nuestras elecciones alimentarias están determinadas por múltiples factores que interactúan a diferentes escalas -individuales, familiares y sociales - y que deben ser contemplados si queremos acercarnos a una dieta más saludable (Leng et al. 2017). A lo anterior se le suma el hecho de que los consumidores se han desconectado y desinteresado cada vez más de los sistemas alimentarios debido al alejamiento progresivo con los actores encargados de la producción. Esta desconexión se ha observado en tres niveles: 1) físico, entre las zonas urbanas donde vive la mayoría de las personas y las zonas rurales donde se producen alimentos; 2) económico, hay más intermediarios entre consumidores y agricultores, con una mayor proporción de valor que es apropiado en la cadena de suministro a expensas de los agricultores; y 3) cognitivo, disminución del conocimiento de cómo se producen y procesan los alimentos (Grace 2016). Como resultado, el hecho de que las elecciones de alimentos tengan implicancias para los sistemas agrícolas se ha vuelto menos obvio y menos importante en la jerarquía de las preocupaciones diarias (Grace 2016). Esta complejidad nos obliga a reflexionar sobre la importancia de desarrollar políticas públicas para direccionar nuestro consumo de alimentos hacia caminos más saludables y sostenibles. En el fondo, se trata de llevar a su producción la lógica de consumidores responsables que ya se da en otras cadenas, y que lleva a que estemos dispuestos a pagar algo más por un producto con huellas ambiental y socialmente más favorables (Fernández 2015).

No es el propósito de este artículo revisar la efectividad del amplio abanico de opciones 
de políticas alimentarias, por lo que nos limitaremos a realizar unos breves comentarios al respecto (para más información ver por ejemplo: Peeters 2018; Mozzafarian et al. 2018; Lusk and McCluskey 2018; Branca et al. 2019). La educación alimentaria y ambiental es un buen primer paso para cambiar el rumbo de las dietas. Más allá de la educación nutricional en las instituciones educativas y las campañas de comunicación, el etiquetado frontal de alimentos es una de las mejores herramientas para que los consumidores reduzcan el consumo de alimentos y nutrientes no deseados (en particular, los de tipo directivo con advertencias o sellos) (Cabrera et al. 2017; Shangguan et al. 2018; FIC 2020). En este sentido, afortunadamente al momento de escribirse este artículo se está discutiendo una ley al respecto en la Argentina. Sin embargo, dado que estamos lejos de ser los agentes racionales que pensamos que somos y que nuestras elecciones alimentarias están fuertemente influenciadas por la cultura popular, la publicidad y los medios de comunicación (Leng et al. 2017; Allemandi et al. 2018), resulta necesario abordar los consumos desde múltiples aristas. Por ejemplo, la evidencia indica que las políticas fiscales (como los impuestos y los subsidios) y su combinación con el etiquetado son una de las medidas más prometedoras para el mejoramiento de la dieta y la prevención de las ECNT (Thow et al. 2018; Taillie et al. 2020). A pesar de la supuesta inelasticidadprecio generalizada, una revisión reciente encontró que la disminución de $10 \%$ del precio se asociaba con un aumento del 14\% en el consumo de frutas y verduras y a un aumento del 16\% del consumo de otros alimentos saludables (Afshin et al. 2017). Las políticas fiscales también han demostrado ser muy efectivas para reducir el consumo de los alimentos insanos: un aumento del 10\% en el precio se asociaba con un $7 \%$ de menor consumo de bebidas azucaradas y un 3\% de menor consumo de comida rápida (Afshin et al. 2017). Respecto a esto último, cabe destacar que la aplicación de impuestos a las bebidas azucaradas y los alimentos ultraprocesados representa un gran desafío debido a la oposición que suelen presentar la industria azucarera y alimentaria, influenciando sobre organismos internacionales y gobiernos con el objetivo de bloquear dichas iniciativas (Stuckler et al. 2016; FIC 2020). Un argumento muy común, utilizado también por la industria del tabaco, es que la aplicación de impuestos afecta de manera desproporcionada a los pobres. Sin embargo, y de la misma manera que ocurrió con el tabaco, los perjuicios sanitarios a mediano y largo plazo son mayores para la sociedad en comparación a los beneficios económicos de corto plazo, particularmente para los pobres, lo que permite que las políticas de regulación de estos alimentos estén avanzando en muchos países del mundo (Falbe and Madsen 2017; Bukhman et al. 2020).

Por otro lado, cuando pensamos en la reducción del consumo interno de los alimentos consumidos en exceso, pueden ocurrir dos situaciones no excluyentes que ilustraremos a través de un ejemplo con la carne vacuna dada su relevancia para el país. En la primera situación, la disminución de la demanda nacional generada por un cambio en la dieta podría impactar directamente sobre el sector productivo causando una disminución en el stock y la producción ganadera. El efecto directo asociado sería la reducción del uso de recursos y de la emisión de contaminantes en el ambiente. Pero la superficie liberada también podría dedicarse a otras alternativas productivas. Por ejemplo, las tierras con aptitud agrícola podrían ser utilizadas para producir cultivos anuales, un fenómeno observado en la región pampeana de nuestro país desde hace varias décadas (Viglizzo et al. 2011). En la segunda situación, la reducción en la demanda del mercado interno podría causar un incremento en el saldo exportable de carne vacuna, generando una oportunidad para que el sector aumente su participación en el mercado internacional y sea una fuente adicional de ingresos de divisas (considerando el consumo recomendado de 14 g/día, o $5 \mathrm{~kg} /$ año, el saldo exportable sería de 2.57 millones de toneladas). Si se levantasen las fuertes restricciones que hoy afectan al mercado de la carne vacuna, las posibilidades de inserción resultarían aun mayores, en tanto se generarían mayores incentivos para incrementar la producción (Britos et al. 2015). Bajo este escenario, el impacto ambiental directo de los habitantes de la Argentina a través de su dieta sería menor (impacto per cápita), pero no así el del sector ganadero en su totalidad, el cual podría aumentar debido a que por el Efecto Rebote posiblemente se fomentaría un mayor desmonte parcial (rolado) o deforestación total para pasturas implantadas (principalmente en la ecorregión chaqueña). A su vez, dicho impacto estará fuertemente determinado por los modelos de producción aplicados. Por ejemplo, no será el mismo impacto si la participación 
del engorde en corral aumenta (como se lo fomentó hace algunos años para mantener los precios internos bajos) que si la alimentación del ganado es predominantemente pastoril, con poca suplementación, y la productividad se fomenta a través del buen manejo del rodeo (Fisher and Bilenca 2020). Aun así, se desconocen los posibles efectos de rebote ligados al aumento de la eficiencia productiva de la industria ganadera. Sin embargo, el interés creciente de la población en relación con los temas éticos vinculados a la producción y el consumo de carnes, además de los ambientales y sanitarios, podría cambiar de manera significativa el escenario global de oferta y demanda de estos alimentos en las próximas décadas (Arneth et al. 2019; Harwatt et al. 2019). Dicho efecto se está manifestando por el aumento en la prevalencia de veganos y vegetarianos, así como el aumento mundial en el número de empresas dedicadas a la producción de carne in-vitro y de substitutos de la carne elaborados a base de materias primas vegetales como Impossible Food y Beyond Burguer (Gerhardt et al. 2019). Sin embargo, se requiere cautela respecto a estos alimentos, ya que aún existen ciertos cuestionamientos referidos a los impactos sobre la salud ( $\mathrm{Hu}$ et al. 2019).

Los desafíos de adoptar una dieta saludable y sostenible en la Argentina - y en cualquier parte del mundo- exceden a los tratados en este artículo y requieren de una aproximación desde múltiples disciplinas, en particular de las ciencias sociales, ya que la pobreza es la principal barrera que tienen las personas para adoptar una dieta saludable. Es innegable que tanto en nuestro país como en el resto del mundo existen problemas intrínsecos al sistema político-económico que afectan al funcionamiento del sistema alimentario y la dieta de las personas, y que también necesitan ser considerados.

\section{Conclusiones}

La necesidad deincrementarla disponibilidad de alimentos a mediados del siglo XX se vio reflejada en la mejora de los rendimientos de los cultivos y los animales gracias a una combinación de mejoras genéticas, prácticas de manejo y sanitarias, y uso de insumos (e.g., fertilizantes, pesticidas, agua, etc.). Después, cambiar las formas de producir alimentos en el campo se presentó como el camino necesario para disminuir las 'huellas' ambientales. Más recientemente, el análisis de los sistemas agroalimentarios tomó perspectivas más amplias e incluyó la reducción de pérdidas y desperdicios para abordar las ineficiencias, y a la adopción de dietas saludables para brindar beneficios ambientales y sanitarios de manera simultánea.

La adopción de dietas saludables y sostenibles representa un gran desafío en nuestro país debido a la actual mala calidad de la dieta argentina y el profundo arraigo cultural hacia algunos alimentos de origen animal que desplazan el consumo de fuentes de proteínas de origen vegetal (e.g., legumbres, cereales y frutos secos). Dadas las actuales condiciones sanitarias y ambientales del país, las dietas saludables representan un camino de tipo win-win, con beneficios para las personas y la naturaleza. Mejorar la dieta en la Argentina implica reducir el consumo de carnes rojas y procesadas, de alimentos ultraprocesados y bebidas azucaradas, e incrementar el consumo de frutas, verduras, frutos secos, legumbres y carne de pescado. Este proceso podría ser interpretado como una invasión cultural que busca homogeneizar las dietas y perder la identidad. Sin embargo, las recomendaciones para adoptar dietas saludables y sostenibles proveen un marco general a partir del cual cada sociedad se adaptará de acuerdo a sus circunstancias y posibilidades. En ese sentido, la transición debería verse como una oportunidad de revalorizar alimentos producidos localmente que han quedado solapados por la oferta de la industria alimentaria, así como para realizar combinaciones novedosas inspiradas en la naturaleza multicultural del país. Reducir el consumo de carnes no debería implicar necesariamente un abandono de prácticas culinarias tradicionales, sino una modificación progresiva de algunas, como por ejemplo limitar el consumo de carne vacuna a ocasiones especiales (como al asado de los domingos) e incluir más alimentos de origen vegetal.

Sorprendentemente, aun con una población dispuesta a adoptar una dieta saludable, el sistema agroalimentario nacional presenta limitaciones importantes para proveer los alimentos que constituyen la 'canasta alimentaria saludable'. Por fortuna, nuestras condiciones agroecológicas proveen un gran potencial para satisfacer dicha demanda y contribuir a la provisión de alimentos saludables al resto del mundo. Por ello, la alineación de las políticas de producción 
agropecuaria y ambientales con las de la alimentación y nutrición humana podría tener beneficios sinérgicos importantes para la población.Sinembargo, esnecesariodesarrollar una mirada integral de la sustentabilidad (i.e., ambiental, productiva y social) a fines de prever el surgimiento de conflictos de intereses e inherentes compromisos entre los aspectos socioeconómicos, productivos y ambientales, ya que es poco probable que los agricultores reconsideren su modelo de producción ni que los consumidores reorienten radicalmente sus patrones de compra sin que ocurra un cambio importante en los incentivos dentro de los sistemas alimentarios.

Junto con las mejoras en la tecnología de los procesos productivos en el campo y en agrologística, la adopción de dietas saludables
- como las recomendadas por las guías alimentarias (GABAs) - representa una estrategiaclaveparaasegurarlasustentabilidad de los sistemas agrolimentarios al ejercer un efecto directo a través de la demanda sobre la cadena productiva, al mismo tiempo que extiende su campo de acción hacia el mejoramiento de la salud pública mediante la prevención (e incluso tratamiento) de las enfermedades crónicas no-transmisibles más importantes.

Agradecimientos. A.D.G. y R.J.F. son investigadores del CONICET, que también financió la beca doctoral de E.M.A. Sebastián Aguiar y tres revisores anónimos aportaron valiosos comentarios y sugerencias que contribuyeron a mejorar sustancialmente el artículo.

\section{ReFerencias}

Adesogan, A. T., A. H. Havelaar, S. L. McKune, M. Eilittä, and G. E. Dahl. 2020. Animal source foods: Sustainability problem or malnutrition and sustainability solution? Perspective matters. Global Food Security 25:00325. https: 7/doi.org/10.1016/j.gfs.2019.100325.

Afshin, A., J. L. Penalvo, L. Del Gobbo, J. Silva, M. Michaelson, et al. 2017. The prospective impact of food pricing on improving dietary consumption: a systematic review and meta-analysis. PloS One 12:e0172277. https://doi.org/ 10.1371/journal.pone.0172277.

Afshin, A., P. J. Sur, K. A. Fay, L. Cornaby, G. Ferrara, et al. 2019. Health effects of dietary risks in 195 countries. 1990-2017: a systematic analysis for the Global Burden of Disease Study 2017. The Lancet 393:1958-1972.

Aguiar, S., M. Texeira, L. A. Garibaldi, and E. G. Jobbágy. 2020. Global changes in crop diversity: Trade rather than production enriches supply. Global Food Security 26:100385. https://doi.org/10.1016/j.gfs.2020.100385.

Aguirre, P. 2017. Una Historia Social de la Comida. Lugar: Buenos Aires.

Aguirre, J. A., M. L. Donato, M. Buscio, V. Ceballos, M. Armeno, L. Aizpurúa, and L. Arpía. 2019. Compromiso neurológico grave por déficit de vitamina B12 en lactantes hijos de madres veganas y vegetarianas. Archivos Argentinos de Pediatría 117:e420-e424. https://doi.org/10.5546/aap.2019.e420.

Albert, J. 2007. Global patterns and country experiences with the formulation and implementation of food-based dietary guidelines. Annals of Nutrition and Metabolism 51:2-7. https://doi.org/10.1159/000103560.

Alexander, P., M. D. Rounsevell, C. Dislich, J. R. Dodson, K. Engström, and D. Moran. 2015. Drivers for global agricultural land use change: The nexus of diet, population, yield and bioenergy. Global Environmental Change 35:138-147. https: //doi.org/10.1016/j.gloenvcha.2015.08.011.

Alexander, P., C. Brown, A. Arneth, J. Finnigan, and M. D. Rounsevell. 2016. Human appropriation of land for food: The role of diet. Global Environmental Change 41:88-98. https://doi.org/10.1016/j.gloenvcha.2016.09.005.

Alexander, P., A. Reddy, C. Brown, R. C. Henry, and M. D. Rounsevell. 2019. Transforming agricultural land use through marginal gains in the food system. Global Environmental Change 57:101932. https://doi.org/10.1016/ j.gloenvcha.2019.101932.

Allemandi, L., L. Castronuovo, M. V. Tiscornia, M. Ponce, and V. Schoj. 2018. Food advertising on Argentinean television: are ultra-processed foods in the lead? Public Health Nutrition 21:238-246. https://doi.org/10.1017/ S1368980017001446.

Andersson D., R. Linscot, and J. Nässén. 2019. Estimating car use rebound effects from Swedish microdata. Energy Efficiency 12:2215-2225. https://doi.org/10.1007/s12053-019-09823-w.

Arneth, A., H. Barbosa, T. Benton, K. Calvin, E. Calvo, S. Connors, and F. Driouech. 2019. IPCC special report on climate change, desertification, land degradation, sustainable land management, food security, and greenhouse gas fluxes in terrestrial ecosystems. Summary for Policy Makers. Geneva, Switzerland Intergovernmental Panel on Climate Change (IPCC).

Arrieta. E. M. 2017. Berretines de verdura. El Gato y La Caja. URL: https://elgatoylacaja.com.ar/berretines-deverdura.

Arrieta, E. M., and A. D. González. 2019. Energy and carbon footprints of food: Investigating the effect of cooking. Sustainable Production and Consumption 19:44-52. https://doi.org/10.1016/j.spc.2019.03.003.

Arrieta, E. M., F. J. Guerrieri, and H. Pizarro. 2019. Glifosanto. El Gato y La Caja. URL: https://elgatoylacaja.com.ar/ glifosanto.

Arrieta, E. M., D. A. Cabrol, A. Cuchietti, and A. D. González. 2020. Biomass consumption and environmental footprints of beef cattle production in Argentina. Agricultural Systems 185:102944. https://doi.org/10.1016/j.agsy.2020.102944.

Bauman, Z. 2011. Daños colaterales: desigualdades sociales en la era global. Fondo de Cultura Económica.

Bechthold, A., H. Boeing, C. Schwedhelm, G. Hoffmann, S. Knüppel, et al. 2019. Food groups and risk of coronary heart disease, stroke and heart failure: a systematic review and dose-response meta-analysis of prospective studies. Critical Reviews in Food Science and Nutrition 59:1071-1090. https://doi.org/10.1080/10408398.2017.1392288. 
Bechthold, A., H. Boeing, I. Tetens, L. Schwingshackl, and U. Nöthlings. 2018. Perspective: food-based dietary guidelines in Europe - scientific concepts, current status, and perspectives. Advances in Nutrition 9:544-560. https://doi.org/ 10.1093/advances/nmy033.

Behrens, P., J. C. Kiefte-de Jong, T. Bosker, J. F. Rodrigues, A. De Koning, and A. Tukker. 2017. Evaluating the environmental impacts of dietary recommendations. Proceedings of the National Academy of Sciences USA 114: 13412-13417. https://doi.org/10.1073/pnas.1711889114.

Berners-Lee, M. 2019. There is no Planet B: a handbook for the make-or-break years. Cambridge University Press. https://doi.org/10.1017/9781108545969.

Borras Jr., S. M., J. C. Franco, S. R. Isakson, L. Levidow, and P. Vervest. 2016. The rise of flex crops and commodities: implications for research. The Journal of Peasant Studies 43:93-115. https://doi.org/10.1080/03066150.2015.1036417.

Bouvard, V., D. Loomis, K. Z. Guyton, Y. Grosse, F. E. Ghissassi, et al. 2015. Carcinogenicity of consumption of red and processed meat. The Lancet Oncology 16:1599-1600. https://doi.org/10.1016/S1470-2045(15)00444-1.

Bouwman, A. F., A. H. Beusen, and G. Billen. 2009. Human alteration of the global nitrogen and phosphorus soil balances for the period 1970-2050. Global Biogeochemical Cycles 23. https://doi.org/10.1029/2009GB003576.

Branca, F., A. Lartey, S. Oenema, V. Aguayo, Stordalen, et al. 2019. Transforming the food system to fight noncommunicable diseases. British Medical Journal 364:I296. https://doi.org/10.1136/bmj.1296.

Bringezu, S., H. Schütz, W. Pengue, M. O’Brien, F. García, et al. 2014. Assessing global land use: balancing consumption with sustainable supply. United Nations Environment Programme.

Britos, S., N. Chichizola, R. Feeney, P. Mac Clay, and F. Vilella. 2015. Comer saludable y exportar seguridad alimentaria al mundo. Buenos Aires. Argentina.

Bukhman, G., A. O. Mocumbi, R. Atun, A. E. Becker, Z. Bhutta, et al. 2020. The Lancet NCDI Poverty Commission: bridging a gap in universal health coverage for the poorest billion. The Lancet 396:991-1044. https://doi.org/10.1016/ S0140-6736(20)31907-3.

Cabrera, M., L. Machín, A. Arrúa, L. Antúnez, M. R. Curutchet, A. Giménez, and G. Ares. 2017. Nutrition warnings as front-of-pack labels: influence of design features on healthfulness perception and attentional capture. Public Health Nutrition 20:3360-3371. https://doi.org/10.1017/S136898001700249X.

Cáceres, D. M. 2015. Accumulation by Dispossession and Socio-Environmental Conflicts Caused by the Expansion of Agribusiness in Argentina. Journal of Agrarian Change 15:116-147. https://doi.org/10.1111/joac.12057.

Carolan, M. 2013. The Real Cost of Cheap Food. Routledge. https://doi.org/10.4324/9781849776776.

CDC. 2020. Evidence used to update the list of underlying medical conditions that increase a person's risk of severe illness from COVID-19. Center for Disease Control and Prevention. Washington D.C., USA.

Céspedes, E. M., and F. B. Hu. 2015. Dietary patterns: from nutritional epidemiologic analysis to national guidelines. American Journal of Clinical Nutrition 101:899-900. https://doi.org/10.3945/ajcn.115.110213.

Chaudhary, A., D. Gustafson, and A. Mathys. 2018. Multi-indicator sustainability assessment of global food systems. Nature Communications 9:848. https://doi.org/10.1038/s41467-018-03308-7.

Clark, A., M. Jit, C. Warren-Gash, B. Guthrie, H. H. Wang, et al. 2020. Global, regional, and national estimates of the population at increased risk of severe COVID-19 due to underlying health conditions in 2020: a modelling study. The Lancet Global Health 8:e1003-e1017.

Clark, M., and D. Tilman. 2017. Comparative analysis of environmental impacts of agricultural production systems, agricultural input efficiency, and food choice. Environmental Research Letters 12:064016. https://doi.org/10.1088/ 1748-9326/aa6cd5.

Clark, M. A., M. Springmann, J. Hill, and D. Tilman. 2019. Multiple health and environmental impacts of foods. Proceedings of the National Academy of Sciences USA 116:23357-23362. https://doi.org/10.1073/pnas.1906908116.

Dahmus, J. B. 2014. Can efficiency improvements reduce resource consumption? A historical analysis of ten activities. Journal of Industrial Ecology 18:883-897. https://doi.org/10.1111/jiec.12110.

Dainese, M., E. A. Martin, M. A. Aizen, M. Albrecht, I. Bartomeus, et al. 2019. A global synthesis reveals biodiversitymediated benefits for crop production. Science Advances 5:eaax0121.

De Vries, M. D., C. E. Van Middelaar, and I. J. M. De Boer. 2015. Comparing environmental impacts of beef production systems: A review of life cycle assessments. Livestock Science 178:279-288. https://doi.org/10.1016/ j.livsci.2015.06.020.

Díaz, S. M., J. Settele, E. Brondízio, H. Ngo, M. Guèze, J. Agard, and K. Chan. 2019. The global assessment report on biodiversity and ecosystem services: Summary for policymakers. Bonn, Germany. Intergovernmental Panel on Biodiversity and Ecosystem Services (IPBES).

Dobermann, A., R. Nelson, D. Beever, D. Bergvinson, E. Crowley, et al. 2013. Solutions for sustainable agriculture and food systems. Sustainable Development Solutions Network.

Echeverria, R. 2020. Fixing the global food system after coronavirus. International Food Policy Research Institute. URL: https://tinyurl.com/y8zh46t2.

Falbe, J., and K. Madsen. 2017. Growing momentum for sugar-sweetened beverage campaigns and policies: costs and considerations. American Journal of Public Health 107:835-838. https://doi.org/10.2105/AJPH.2017.303805.

Fang, H., J. Kang, and D. Zhang. 2017. Microbial production of vitamin B12: a review and future perspectives. Microbial Cell Factories 16:15. https://doi.org/10.1186/s12934-017-0631-y.

Fanzo, J., C. Hawkes, E. Udomkesmalee, A. Afshin, L. Allemandi, et al. 2018. Global Nutrition Report: Shining a light to spur action on nutrition. Bristol, UK: Development Initiatives.

FAO. 2017. The future of food and agriculture - Trends and challenges. Food and Agriculture Organization. Rome. Italy.

FAO. 2018. The future of food and agriculture - Alternative pathways to 2050. Food and Agriculture Organization. Rome. Italy.

FAO. 2019. The State of Food Security and Nutrition in the World 2019. Safeguarding against economic slowdowns and downturns. Food and Agriculture Organization. Rome. Italy. 
FAO. 2020. Food-based dietary guidelines. Rome. FAO. URL: https://tinyurl.com/yxhspneu.

FAO/WHO. 1992. International conference on nutrition: World Declaration and Plan of Action for Nutrition. FAO/ WHO. Rome. Italy.

FAOSTAT. 2020. Statistical databases. Food and Agriculture Organization. URL: http://www.fao.org/faostat/en/.

Fardet, A., and Y. Boirie. 2014. Associations between food and beverage groups and major diet-related chronic diseases: an exhaustive review of pooled/meta-analyses and systematic reviews. Nutrition Reviews 72:741-762. https://doi.org/ 10.1111/nure.12153.

Felcman, I. L. 2013. Plan Estratégico Agroalimentario y Agroindustrial Participativo y Federal. 2010-2020 (PEA²). Ministerio de Agricultura. Ganadería y Pesca. Buenos Aires. Argentina.

Fernández, R. J. 2015. Las verdades más incómodas del cambio climático no son climáticas. Ecología Austral 25:149-157. https://doi.org/10.25260/EA.15.25.2.0.160.

Fernández, R. J., P. Rush, and M. C. Plencovich. 2019. Agroecología y agricultura industrial: ¿dos culturas irreconciliables? Agronomía y Ambiente 39:68-94.

Feskens, E. J., D. Sluik, and G. J. van Woudenbergh. 2013. Meat consumption, diabetes, and its complications. Current Diabetes Reports 13:298-306. https://doi.org/10.1007/s11892-013-0365-0.

FIC. 2020. Políticas para promover un etiquetado frontal en alimentos y bebidas: recomendaciones para Argentina. Fundación Interamericana del Corazón. Buenos Aires, Argentina.

Fischer, C. G., and D. Bilenca. 2020. Can we produce more beef without increasing its environmental impact? Argentina as a case study. Perspectives in Ecology and Conservation 18:1-11. https://doi.org/10.1016/j.pecon.2019.12.002.

Fischer, C. G., and T. Garnett. 2016. Plates. pyramids. and planets: developments in national healthy and sustainable dietary guidelines: a state of play assessment. Food and Agriculture Organization of the United Nations and the Food Climate Research Network (University of Oxford).

Foley, J. A., N. Ramankutty, K. A. Brauman, E. S. Cassidy, J. S. Gerber, et al. 2011. Solutions for a cultivated planet. Nature 478:337-342. https://doi.org/10.1038/nature10452.

Fraser, E., A. Legwegoh, K. C. Krishna, M. CoDyre, M., G. Dias, et al. 2016. Biotechnology or organic? Extensive or intensive? Global or local? A critical review of potential pathways to resolve the global food crisis. Trends in Food Science and Technology 48:78-87. https://doi.org/10.1016/j.tifs.2015.11.006.

Freeman, R., M. Yearworth, and C. Preist. 2016. Revisiting Jevons' paradox with system dynamics: Systemic causes and potential cures. Journal of Industrial Ecology 20:341-353. https://doi.org/10.1111/jiec.12285.

García-López, G. A., and N. Arizpe. 2010. Participatory processes in the soy conflicts in Paraguay and Argentina. Ecological Economics 70:196-206. https://doi.org/10.1016/j.ecolecon.2010.06.013.

Gallo, D., M. Manuzza, N. Echegaray, J. Montero, M. Munner, et al. 2015. Alimentación vegetariana. Grupo de Trabajo de Alimentos. Sociedad Argentina de Nutrición.

Garibaldi, L. A., G. Andersson, C. F. Ferrari, and N. Pérez-Méndez. 2018. Seguridad alimentaria. medio ambiente y nuestros hábitos de consumo. Ecología Austral 28:572-580. https://doi.org/10.25260/EA.18.28.3.0.768.

Garibaldi, L. A., N. Pérez-Méndez, M. P. Garratt, B. Gemmill-Herren, F. E. Miguez, and L. V. Dicks. 2019. Policies for ecological intensification of crop production. Trends in Ecology and Evolution 34:282-286. https://doi.org/10.1016/ j.tree.2019.01.003.

Gerhardt, C., G. Suhlmann, F. Ziemßen, D. Donnan, M. Warschun, and H. Kühnle. 2019. How Will Cultured Meat and Meat Alternatives Disrupt the Agricultural and Food Industry? ATKearney. URL: https://tinyurl.com/y2ek26tw.

Gliessman, S. R. 2007. Agroecology: The Ecology of Sustainable Food Systems. CRC Press. https://doi.org/10.1201/ b17420.

Godfray, H. C. J., and T. Garnett. 2014. Food security and sustainable intensification. Philosophical Transactions of the Royal Society B: Biological Sciences 369:20120273. https://doi.org/10.1098/rstb.2012.0273.

Godfray, H. C. J., P. Aveyard, T. Garnett, J. W. Hall, T. J. Key, et al. 2018. Meat consumption. health. and the environment. Science 361:eaam5324. https://doi.org/10.1126/science.aam5324.

Godlee, F. 2020. COVID-19: What we eat matters all the more now. British Medical Journal 370:m2840. https://doi.org/ 10.1136/bmj.m2840.

González, A. D., B. Frostell, and A. Carlsson-Kanyama. 2011. Protein efficiency per unit energy and per unit greenhouse gas emissions: potential contribution of diet choices to climate change mitigation. Food Policy 36:562-570. https: \%/doi.org/10.1016/j.foodpol.2011.07.003.

Gordillo, G., and O. M. Jerónimo. 2013. Seguridad y soberanía alimentaria: documento base para la discusión. Organización de las Naciones Unidas para la Alimentación y la Agricultura. Roma. Italia.

Grace, D. 2016. Influencing food environments for healthy diets through food safety. Food and Agriculture Organization. Rome, Italy.

Gras, C., and D. M. Cáceres. 2020. Technology, nature's appropriation and capital accumulation in modern agriculture. Current Opinion in Environmental Sustainability 45:1-9. https://doi.org/10.1016/j.cosust.2020.04.001.

Gustavsson, J., C. Cederberg, U. Sonesson, R. Van Otterdijk, and A. Meybeck. 2011. Global food losses and food waste. Food and Agriculture Organization. Rome, Italy.

Hamant, O. 2020. Plant scientists can't ignore Jevons paradox anymore. Nature Plants 6:720-722. https://doi.org/10.1038/ s41477-020-0722-3.

Herrero, M., P. K. Thornton, A. M. Notenbaert, S. Wood, S. Msangi, et al. 2010. Smart investments in sustainable food production: revisiting mixed crop-livestock systems. Science 327:822-825. https://doi.org/10.1126/science.1183725.

Herrero, M., S. Wirsenius, B. Henderson, C. Rigolot, P. Thornton, et al. 2015. Livestock and the environment: what have we learned in the past decade? Annual Review of Environment and Resources 40:177-202. https://doi.org/10.1146/ annurev-environ-031113-093503.

Holt-Giménez, E., A. Shattuck, M. Altieri, H. Herren, and S. Gliessman. 2012. We already grow enough food for 10 billion people... and still can't end hunger. Journal of Sustainable Agriculture 36:595-598. https://doi.org/10.1080/ 10440046.2012.695331. 
Hu, F. B., B. O. Otis, and G. McCarthy. 2019. Can Plant-Based Meat Alternatives Be Part of a Healthy and Sustainable Diet? Journal of the American Medical Association 322:1547-1548. https://doi.org/10.1001/jama.2019.13187.

IATF. 2020. Joint statement on noncommunicable diseases and COVID-19. Inter-American Task Force on Noncommunicable Diseases. Pan American Health Organization. Washington, D.C., United States.

IFPRI. 2017. Global food policy report. International Food Policy Research Institute. Washington. DC, USA.

IPCC. 2019. Refinement of the 2006 IPCC Guidelines for National Greenhouse Gas Inventories. Intergovernmental Panel on Climate Change. National Greenhouse Gas Inventories Program. IGES. Japan.

Jaurena, G. 2019. Capítulo 4: Los cambios en los sistemas de medición en ganadería —la tensión entre productividad y ambiente- - . Revista Argentina de Producción Animal 39:77-86.

Kastner, T., M. J. I. Rivas, W. Koch, and S. Nonhebel. 2012. Global changes in diets and the consequences for land requirements for food. Proceedings of the National Academy of Sciences USA 109:6868-6872. https://doi.org/10.1073/ pnas.1117054109.

Keating, B. A., M. Herrero, P. S. Carberry, J. Gardner, and M. B. Cole. 2014. Food wedges: framing the global food demand and supply challenge towards 2050. Global Food Security 3:125-132. https://doi.org/10.1016/j.gfs.2014.08.004.

Koeth, R. A., Z. Wang, B. S. Levison, J. A. Buffa, E. Org, et al. 2013. Intestinal microbiota metabolism of L-carnitine, a nutrient in red meat, promotes atherosclerosis. Nature Medicine 19:576. https://doi.org/10.1038/nm.3145.

Krausmann, F. and E. Langthaler. 2019. Food regimes and their trade links: A socio-ecological perspective. Ecological Economics 160:87-95. https://doi.org/10.1016/j.ecolecon.2019.02.011.

Larsson, S. C., and N. Orsini. 2014. Red meat and processed meat consumption and all-cause mortality: a meta-analysis. American Journal of Epidemiology 179:282-289. https://doi.org/10.1093/aje/kwt261.

Leclère, D., M. Obersteiner, M. Barrett, S. H. Butchart, A. Chaudhary, et al. 2020. Bending the curve of terrestrial biodiversity needs an integrated strategy. Nature 585:551-556.

Li, Y., J. Schoufour, D. D. Wang, K. Dhana, A. Pan, et al. 2020. Healthy lifestyle and life expectancy free of cancer, cardiovascular disease, and type 2 diabetes: prospective cohort study. British Medical Journal 368:16669. https: //doi.org/10.1136/bmj.16669.

Liere, H. S. Jha, and S. M. Philpott. 2017. Intersection between biodiversity conservation, agroecology, and ecosystem services. Agroecology and Sustainable Food Systems 41:723-760. https://doi.org/10.1080/21683565.2017.1330796.

Lusk, J. L., and J. McCluskey. 2018. Understanding the impacts of food consumer choice and food policy outcomes. Applied Economic Perspectives and Policy 40:5-21. https://doi.org/10.1093/aepp/ppx054.

McIntyre, B. D., H. R. Herren, J. Wakhungu, and R. T. Watson. 2009. Agriculture at a Crossroads: IAASTD Global Report. Agriculture at a Crossroads: IAASTD Global Report.

Magliano, D. J., V. H. Loh, J. L. Harding, J. Botton, and J. E. Shaw. 2014. Persistent organic pollutants and diabetes: a review of the epidemiological evidence. Diabetes and Metabolism 40:1-14. https://doi.org/10.1016/j.diabet.2013.09.006.

Mason-D'Croz, D., J. R. Bogard, T. B. Sulser, N. Cenacchi, S. Dunston, M. Herrero, and K. Wiebe. 2019. Gaps between fruit and vegetable production. demand. and recommended consumption at global and national levels: an integrated modelling study. The Lancet Planetary Health 3:e318-e329. https://doi.org/10.1016/S2542-5196(19)30095-6.

MAyDS. 2020. Informe del estado del ambiente 2019. Ministerio de Ambiente y Desarrollo Sustentable. Buenos Aires. Argentina.

Melina, V., W. Craig, and S. Levin. 2016. Position of the Academy of Nutrition and Dietetics: vegetarian diets. Journal of the Academy of Nutrition and Dietetics 116:1970-1980. https://doi.org/10.1016/j.jand.2016.09.025.

Micha, R. D., S. K. Wallace, and D. Mozaffarian. 2010. Red and processed meat consumption and risk of incident coronary heart disease, stroke, and diabetes mellitus. Circulation 121:2271-2783. https://doi.org/10.1161/CIRCULA TIONAHA.109.924977.

Ministerio de Salud. 2016. Guías Alimentarias para la Población Argentina. Buenos Aires. Argentina.

Monteiro, C. A., G. Cannon, R. B. Levy, J. C. Moubarac, M. L. Louzada, F. Rauber, et al. 2019. Ultra-processed foods: what they are and how to identify them. Public Health Nutrition 22:936-941. https://doi.org/10.1017/S1368980018003762.

Moshiri S., and K. Aliyev. 2017. Rebound effect of efficiency improvement in passenger cars on gasoline in Canada. Ecological Economics 131:330-341. https://doi.org/10.1016/j.ecolecon.2016.09.018.

MSyDS. 2019. Cuarta Encuesta Nacional de Factores de Riesgo. Ministerio de Salud y Desarrollo Social. Buenos Aires. Argentina.

Mozaffarian, D., S. Y. Angell, T. Lang, and J. A. Rivera. 2018. Role of government policy in nutrition-barriers to and opportunities for healthier eating. British Medical Journal 361:k2426. https://doi.org/10.1136/bmj.k2426.

Notarnicola, B., S. Sala, A. Anton, S. J. McLaren, E. Saouter, and U. Sonesson. 2017. The role of life cycle assessment in supporting sustainable agri-food systems: a review of the challenges. Journal of Cleaner Production 140:399-409. https://doi.org/10.1016/j.jclepro.2016.06.071.

PAHO. 2019. Ultra-processed food and drink products in Latin America: Sales. sources. nutrient profiles. and policy implications. Pan American Health Organization. Washington. D.C., USA.

Parrique T., J. Barth, F. Briens, C. Kerschner, A. Kraus-Polk, A. Kuokkanen, J. H. Spangenberg. 2019. Decoupling debunked: Evidence and arguments against green growth as a sole strategy for sustainability. European Environmental Bureau.

Pauly, D., and D. Zeller. 2017. Comments on FAOs state of world fisheries and aquaculture (SOFIA 2016). Marine Policy 77:176-181. https://doi.org/10.1016/j.marpol.2017.01.006.

Pellegrini, P., and R. J. Fernández. 2018. Crop intensification. land use. and on-farm energy-use efficiency during the worldwide spread of the green revolution. Proceedings of the National Academy of Sciences USA 115:2335-2340. https://doi.org/10.1073/pnas.1717072115.

Pelletier, N., E. Audsley, S. Brodt, T. Garnett, P. Henriksson, et al. 2011. Energy intensity of agriculture and food systems. Annual Review of Environment and Resources 36:223-246. https://doi.org/10.1146/annurev-environ-081710161014.

Perfecto, I., and J. Vandermeer. 2012. Separación o integración para la conservación de biodiversidad: la ideología 
detrás del debate "land-sharing" frente a "land-sparing". Ecosistemas 21:180-191.

Persson, U. M., S. Henders, and C. Cederberg. 2014. A method for calculating a land-use change carbon footprint (LUC-CFP) for agricultural commodities-applications to Brazilian beef and soy, Indonesian palm oil. Global Change Biology 20:3482-3491. https://doi.org/10.1111/gcb.12635.

Peeters, A. 2018. Obesity and the future of food policies that promote healthy diets. Nature Reviews Endocrinology 14:430-437. https://doi.org/10.1038/s41574-018-0026-0.

Picasso, V. D., P. D. Modernel, G. Becoña, L. Salvo, L. Gutiérrez, and L. Astigarraga. 2014. Sustainability of meat production beyond carbon footprint: a synthesis of case studies from grazing systems in Uruguay. Meat Science 98: 346-354. https://doi.org/10.1016/j.meatsci.2014.07.005.

Pingali, P. L. 2012. Green revolution: impacts. limits. and the path ahead. Proceedings of the National Academy of Sciences USA 109:12302-12308. https://doi.org/10.1073/pnas.0912953109.

Pollan, M. 2008. In defense of food: An eater's manifesto. Penguin.

Poore. J., and T. Nemecek. 2018. Reducing food's environmental impacts through producers and consumers. Science 360:987-992. https://doi.org/10.1126/science.aaq0216.

Popkin, B. M., L. S. Adair, and S. W. Ng. 2012. Global nutrition transition and the pandemic of obesity in developing countries. Nutrition Reviews 70:3-21. https://doi.org/10.1111/j.1753-4887.2011.00456.x.

Popkin, B. M. 2017. Relationship between shifts in food system dynamics and acceleration of the global nutrition transition. Nutrition Reviews 75:73-82. https://doi.org/10.1093/nutrit/nuw064.

Potts, S. G., V. Imperatriz-Fonseca, H. T. Ngo, M. A. Aizen, J. C. Biesmeijer, et al. 2016. Safeguarding pollinators and their values to human well-being. Nature 540:220-229. https://doi.org/10.1038/nature20588.

Qian, F., M. C. Riddle, J. Wylie-Rosett, and F. B. Hu. 2020. Red and processed meats and health risks: How strong is the evidence? Diabetes Care 43:265-271. https://doi.org/10.2337/dci19-0063.

Röös, E., M. Patel, J. Spångberg, G. Carlsson, and L. Rydhmer. 2016. Limiting livestock production to pasture and byproducts in a search for sustainable diets. Food Policy 58:1-13. https://doi.org/10.1016/j.foodpol.2015.10.008.

Roser, M., and H. Ritchie. 2019. Food per Person. Our World In Data. URL: https://ourworldindata.org/food-perperson.

Ruzzenenti, F., R. Galvin, S. R. Sorrell, D. Font Vivanco, A. Wagner, and H. J. Walnum. 2019. The rebound effect and the Jevon's Paradox: beyond the conventional wisdom. Frontiers in Energy Research 7:90. https://doi.org/10.3389/ fenrg.2019.00090.

Santarelli, R. L., F. Pierre, and D. E. Corpet. 2008. Processed meat and colorectal cancer: a review of epidemiologic and experimental evidence. Nutrition and Cancer 60:131-144. https://doi.org/10.1080/01635580701684872.

Schein, L. 2018. Argentina: Estudio Pérdidas y desperdicios de alimentos vinculado al Objetivo Desarrollo Sostenible para garantizar producción y consumo responsable (ODS 12). Secretaría de Agroindustria. Ministerio de Producción y Trabajo de la Nación. Buenos Aires. Argentina.

Schramski, J. R., C. B. Woodson, and J. H. Brown. 2020. Energy use and the sustainability of intensifying food production. Nature Sustainability 3:257-259. https://doi.org/10.1038/s41893-020-0503-z.

Schwingshackl, L., C. Schwedhelm, G. Hoffmann, A. M. Lampousi, S. Knüppel, et al. 2017a. Food groups and risk of all-cause mortality: a systematic review and meta-analysis of prospective studies. The American Journal of Clinical Nutrition 105:1462-1473. https://doi.org/10.3945/ajcn.117.153148.

Schwingshackl, L., G. Hoffmann, A. M. Lampousi, S. Knüppel, K. Iqbal, et al. 2017b. Food groups and risk of type 2 diabetes mellitus: a systematic review and meta-analysis of prospective studies. European Journal Epidemiology 32: 363-375. https://doi.org/10.1007/s10654-017-0246-y.

Schwingshackl, L., C. Schwedhelm, G. Hoffmann, S. Knüppel, K. Iqbal, et al. 2017c. Food groups and risk of hypertension: a systematic review and dose-response meta-analysis of prospective studies. Advances in Nutrition 8:793-803. https: //doi.org/10.3945/an.117.017178.

Schwingshackl, L., C. Schwedhelm, G. Hoffmann, S. Knüppel, A. Laure Preterre, et al. 2018. Food groups and risk of colorectal cancer. International Journal of Cancer 142:1748-1758. https://doi.org/10.1002/ijc.31198.

Schwingshackl, L., C. Schwedhelm, G. Hoffmann, and H. Boeing. 2019. Potatoes and risk of chronic disease: A systematic review and dose-response meta-analysis. European Journal of Nutrition 58:2243-2251. https://doi.org/10.1007/s00394018-1774-2.

Shah, S. V., and V. A. Fonseca. 2011. Iron and diabetes revisited. Diabetes Care 34:1676-1677. https://doi.org/10.2337/ dc11-0700.

Shangguan, S., A. Afshin, M. Shulkin, W. Ma, D. Marsden, et al. 2019. A meta-analysis of food labeling effects on consumer diet behaviors and industry practices. American Journal of Preventive Medicine 56:300-314. https://doi.org/ 10.1016/j.amepre.2018.09.024.

Silva, L., E. N. Longo, and A. Lopresti. 2003. Guías alimentarias: Guías alimentarias: manual de multiplicadores. Buenos Aires: Asociación Argentina de Dietistas y Nutricionistas Dietistas.

Silvetti, F., G. Soto, D. M. Cáceres, and D. Cabrol. 2013. ¿Por qué la legislación no protege los bosques nativos de Argentina? Conflictos socioambientales y políticas públicas. Mundo Agrario 13.

Siri-Tarino, P. W., Q. Sun, F. B. Hu, and R. M. Krauss. 2010. Saturated fatty acids and risk of coronary heart disease: modulation by replacement nutrients. Current Atherosclerosis Reports 12:384-390. https://doi.org/10.1007/s11883010-0131-6.

Slipzuk, M. 2019. ¿La Argentina produce alimentos para 400 millones de personas? Chequeado. URL: https://inyurl.com/ yanxb569.

Small, D. M., and A. G. DiFeliceantonio. 2019. Processed foods and food reward. Science 363:346-347. https://doi.org/ $10.1126 /$ science.aav0556.

Smith, J., K. Sones, D. Grace, S. MacMillan, S. Tarawali, and M. Herrero. 2013. Beyond milk. meat. and eggs: Role of livestock in food and nutrition security. Animal Frontiers 3:6-13. https://doi.org/10.2527/af.2013-0002.

Springmann, M., H. C. J. Godfray, M. Rayner, and P. Scarborough, P. 2016. Analysis and valuation of the health and 
climate change cobenefits of dietary change. Proceedings of the National Academy of Sciences USA 113:4146-4151. https://doi.org/10.1073/pnas.1523119113.

Springmann, M., M. Clark, D. Mason-D'Croz, K. Wiebe, B. L. Bodirsky, et al. 2018. Options for keeping the food system within environmental limits. Nature 562:519-525. https://doi.org/10.1038/s41586-018-0594-0.

Springmann, M., L. Spajic, M. A. Clark, J. Poore, A. Herforth, et al. 2020. The healthiness and sustainability of national and global food based dietary guidelines: modelling study. British Medical Journal 370:m2322. https://doi.org/ 10.1136/bmj.m2322.

Stevenson, J. R., N. Villoria, D. Byerlee, T. Kelley, and M. Maredia. 2013. Green Revolution research saved an estimated 18 to 27 million hectares from being brought into agricultural production. Proceedings of the National Academy of Sciences USA 110:8363-8368. https://doi.org/10.1073/pnas.1208065110.

Stuckler, D., A. Reeves, R. Loopstra, and M. McKee. 2016. Textual analysis of sugar industry influence on the World Health Organization's 2015 sugars intake guideline. Bulletin of the World Health Organization 94:566. https://doi.org/ 10.2471/BLT.15.165852.

Swannell, R., M. F. Hall, R. Tay, and T. Quested. 2019. The Food Waste Atlas: An important tool to track food loss and waste and support the creation of a sustainable global food system. Resources, Conservation and Recycling 146:534535. https://doi.org/10.1016/j.resconrec.2019.02.006.

Taillie, L. S., M. Reyes, M. A. Colchero, B. Popkin, and C. Corvalán. 2020. An evaluation of Chile's Law of Food Labeling and Advertising on sugar-sweetened beverage purchases from 2015 to 2017: a before-and-after study. PLoS Medicine 17:e1003015. https://doi.org/10.1371/journal.pmed.1003015.

Thornton, P. K. 2010. Livestock production: recent trends. future prospects. Philosophical Transactions of the Royal Society B: Biological Sciences 365:2853-2867. https://doi.org/10.1098/rstb.2010.0134.

Thow, A. M., S. M. Downs, C. Mayes, H. Trevena, T. Waqanivalu, et al. 2018. Fiscal policy to improve diets and prevent non communicable diseases: from recommendations to action. Bulletin of the World Health Organization 96:201-210. https://doi.org/10.2471/BLT.17.195982.

Tilman, D., C. Balzer, J. Hill, and B. L. Befort. 2011. Global food demand and the sustainable intensification of agriculture. Proceedings of the National Academy of Sciences USA 108:20260-20264. https://doi.org/10.1073/pnas.1116437108.

Tittonell, P. 2014. Ecological intensification of agriculture-sustainable by nature. Current Opinion in Environmental Sustainability 8:53-61. https://doi.org/10.1016/j.cosust.2014.08.006.

van Boeckel, T. P., C. Brower, M. Gilbert, B. T. Grenfell, S. A. Levin, et al. 2015. Global trends in antimicrobial use in food animals. Proceedings of the National Academy of Sciences USA 112:5649-5654. https://doi.org/10.1073/ pnas.1503141112.

van Boeckel, T. P., J. Pires, R. Silvester, C. Zhao, J. Song, et al. 2019. Global trends in antimicrobial resistance in animals in low-and middle-income countries. Science 365:eaaw1944. https://doi.org/10.1126/science.aaw1944.

van Zanten, H. H., M. Herrero, O. Van Hal, E. Röös, A. Muller, et al. 2018. Defining a land boundary for sustainable livestock consumption. Global Change Biology 24:4185-4194. https://doi.org/10.1111/gcb.14321.

Vermeulen, S. J., B. M. Campbell, and J. S. Ingram. 2012. Climate change and food systems. Annual Review of Environment and Resources 37:195-222.

Vermeulen, S., T. Park, C. K. Khoury, J. Mockshell, C. Béné, et al. 2019. Changing diets and transforming food systems. Working paper № 282. CGIAR Research Program on Climate Change, Agriculture and Food Security (CCAFS).

Viglizzo, E. F., F. C. Frank, L. V. Carreño, E. G. Jobbágy, H. Pereyra, et al. F. 2011. Ecological and environmental footprint of 50 years of agricultural expansion in Argentina. Global Change Biology 17:959-973. https://doi.org/10.1111/j.13652486.2010.02293.X.

Wang, X., X. Lin, Y. Y. Ouyang, J. Liu, G. Zhao, A. Pan, and F. B. Hu. 2016. Red and processed meat consumption and mortality: dose-response meta-analysis of prospective cohort studies. Public Health Nutrition 19:893-905. https: //doi.org/10.1017/S1368980015002062.

Wang, D. D., Y. Li, A. Afshin, M. Springmann, D. Mozaffarian, et al. 2019. Global improvement in dietary quality could lead to substantial reduction in premature death. The Journal of Nutrition 149:1065-1074. https://doi.org/10.1093/jn/ nxz010.

WCRF/AICR. 2018. Diet, Nutrition, Physical Activity and Cancer: a Global Perspective. Continuous Update Project. Expert Report 2018. World Cancer Research Fund/American Institute for Cancer Research.

Hajer, M. A., H. Westhoek, J. Ingram, S. van Berkum, L. Özay. 2016. Food Systems and Natural Resources. United Nations Environment Programme.

Wezel, A., G. Soboksa, S. McClelland, F. Delespesse, and A. Boissau. 2015. The blurred boundaries of ecological. sustainable. and agroecological intensification: a review. Agronomy for Sustainable Development 35:1283-1295. https: //doi.org/10.1007/s13593-015-0333-y.

Willett, W. 2013. Nutritional Epidemiology. New York: Oxford University Press. $3^{\text {rd }}$ Edition.

Willett, W. C., and M. J. Stampfer. 2013. Current evidence on healthy eating. Annual Review of Public Health 34:77-95. https://doi.org/10.1146/annurev-publhealth-031811-124646.

Willett, W., J. Rockström, B. Loken, M. Springmann, T. Lang, et al. 2019. Food in the Anthropocene: the EAT-Lancet Commission on healthy diets from sustainable food systems. The Lancet 393:447-492. https://doi.org/10.1016/S01406736(18)31788-4.

Zoncu, R., A. Efeyan, and D. M. Sabatini. 2011. mTOR: from growth signal integration to cancer, diabetes and ageing. Nature Reviews in Molecular Cell Biology 12:21-35. https://doi.org/10.1038/nrm3025. 